\title{
A CHECKLIST OF THE FAMILY Ichneumonidae (Hymenoptera: Ichneumonoidea) FROM VIETNAM
}

\author{
Pham Thi Nhi*, Khuat Dang Long \\ Institute of Ecology and Biological Resources, VAST
}

\begin{abstract}
The ichneumonid fauna of Vietnam has been studied since the early $20^{\text {th }}$ century, but records of this large insect group were published in scattered papers. In this paper ichneumonid wasps from Vietnam are reviewed and a checklist of published species with local and world distribution is provided. Up to now, 246 ichneumonid species in 87 genera of 18 subfamilies have been recorded from Vietnam. Of this number, one genus and no fewer than 70 species were described on the basis of material collected from Vietnam. In the checklist, valid names, first citation, holotype deposition and synonyms that used previously in Vietnam are provided. In addition, ten species are recorded for the first time from Vietnam, viz. Acerataspis clavata (Uchida, 1934); Charops brachypterus (Cameron, 1897); Chlorocryptus purpuratus (Smith, 1852); Chrionota townesi Uchida, 1957; Enicospilus hirayamai Uchida, 1955; Enicospilus melanocarpus Cameron, 1905; Lathrolestes roerichi Reschikov, 2011; Lytarmes maculipennis (Smith, 1858); Sachtlebenia sexmaculata Townes, 1963 and Stenichneumon appropinquans (Cameron, 1897). Specimens of the aforementioned ten species are currently deposited at Institute of Ecology and Biological Resources (IEBR), Ha Noi, Vietnam. Additionally, host information is available for 25 species that are parasitoids of agricultural pests. This paper provides useful information for further studies on one of the largest hymenopteran families of Vietnam.
\end{abstract}

Keywords: Ichneumonidae, beneficial insects, distribution, new record, parasitoids, Vietnam.

Citation: Pham Thi Nhi, Khuat Dang Long, 2016. A checklist of the family Ichneumonidae (Hymenoptera: Ichneumonoidea) from Vietnam. Tap chi Sinh hoc, 38(4): 411-441. DOI: 10.15625/0866-7160/v38n4.8883.

*Corresponding author: ptnhi2@yahoo.com.

Received 29 October 2016, accepted 20 December 2016

\section{INTRODUCTION}

Ichneumonidae is the largest family within the order Hymenoptera. They are considered to be a beneficial insect group because they are important parasitoids of other invertebrates; common hosts are larvae and pupae of Coleoptera, Hymenoptera, and Lepidoptera. Ichneumonidae is a clearly defined monophyletic group of hymenopteran parasitoids (Sharkey \& Wahl, 1992). Worldwide it probably includes more than 100,000 species of which more than 60,500 are currently described and classified into 48 subfamilies ( $\mathrm{Yu}$ et al., 2012). The development of agriculture and forestry raises problems with insect pests, and ichneumonids are likely to increase in economic importance. However, the role of ichneumonids can only be assessed when the fauna is better known and studied (Gauld, 1991).

The ichneumonid fauna of Vietnam is certainly diverse but poorly studied as yet. The first ichneumonids were reported from Vietnam in the early $20^{\text {th }}$ century (e.g. Krieger, 1906, 1914; Garthwaite \& Desai, 1939) but have mostly been recorded since the 1970s: Townes \& Chiu (1970), Kamath \& Gupta (1972), Gupta \& Tikar (1976), Plant Protection Research Institute (1976), Gauld \& Mitchell (1981), Gupta \& Saxena (1987), Bui (1990), Vu (1992), Pham (1997), Bennet (2001), Kasparyan (1999, 2008), Pham (2004), Pham (2007), Khuat \& Pham (2007), Pham \& Le (2007), Pham et al. (2010, 2011, 2012, 2013), Khalaim (2011), Riedel (2011, 2013), Takasuka et al. (2012), Broad (2014), Reshchikov et al. (2014), Varga et al. (2014), Pham \& van Achterberg (2015).

\section{MATERIALS AND METHODS}

In order to facilitate further studies of the fauna of Vietnamese ichneumonid wasps, a 
checklist of Ichneumonidae from Vietnam is presented for the first time. In this checklist beside the valid name of each species, the following information is also given: the original cobination, citation of the original description and the depository of the holotype, synonyms previously used in Vietnam, distribution in Vietnam, and global distributions, which taken from Taxapad (Yu et al., 2012). Within-country distribution of species is followed the order of areas, provinces (in brackets) from north to south, and outside Vietnam distribution of species follows in the alphabetical order. An asterisk in blankets indicates species newly recorded for the fauna of Vietnam.

Abbreviations are used in thechecklist as follows: AMNH: USA, American Museum of Natural History; ANIC: Australia, Australian Capital Territory, Canberra City, CSIRO, Australian National Insect Collection; BMNH: United Kingdom, London, The Natural History Museum; BPBM: USA, Hawaii, Honolulu, Bernice P. Bishop Museum; CAS: USA, California, San Francisco, California Academy of Sciences; CMNH: China, Shanghai, Museum of Natural History; CNC: Canada, Ontario, Ottawa, Canadian National Collection of Insects; DEI: Germany, Müncheberg, Senckenberg Deutsches Entomologisches Institut; DEZC: Italy, Torino, Dipartimento di Entomologia e Zoologia Applicate all'Ambiente "Carlo Vidano"; DZCU: India, West Bengal, Calcutta, Calcutta University; EIHU: Japan, Hokkaido, Sapporo, Hokkaido University; GPTA: India, Delhi, Delhi University, Department of Zoology, Gupta Collection; HMUG: United Kingdom, Glasgow, Glasgow University, Hunterian Museum; HNHM: Hungary, Budapest, Hungarian Natural History Museum; IFRS: India, Uttar Pradesh, Dehra Dun, Indian Forest Research Institute; IEBR: Vietnam, Ha Noi, Institute of Ecology and Biological Resources; IRSNB: Belgium, Brussels, Institut Royal des Sciences Naturelles de Belgique; IZCAS: China, Beijing, Chinese Academy of Sciences, Institute of Zoology; KUEC: Japan, Fukuoka, Kyushu University; MCCI: Italy, Carmagnola, Museo Civico di Storia Naturale; MLUH: Germany, Halle
(Saale),

Martin-Luther-Universität, Wissenschaftsbereich Zoologie; MNHB: Germany, Berlin, Museum für Naturkunde der Humboldt Universität; MNHN: France, Paris, Muséum National d'Histoire Naturelle; MSNG: Italy, Genova, Museo di Storia Naturale "Giacomo Doria"; MUC: India, Aurangabad, Dr. Babasaheb Ambedkar Marathwada University; MVMA: Australia, Victoria, Museum of Victoria; MZPW: Poland, Warsaw, Polish Academy of Science, Museum of the Institute of Zoology; NHRS: Sweden, Stockholm, Naturhistoriska riksmuseet; NP: National Park; NR: Nature Reserve;OMNH: Japan, Osaka, Osaka Museum of Natural History; OUMNH: United Kingdom, Oxford, University Museum of Natural History; PPRI: Plant Protection Research Institute (Vietnam); RMNH: The Netherlands, Leiden, Centre for Biodiversity Naturalis; ROME: Canada, Royal Ontario Museum; TARI: Taiwan, Taichung, Taiwan Agricultural Research Institute; USNM: USA, Washington D.C., National Museum of Natural History; USU: USA, Logan, Utah State University; UUZM: Sweden, Uppsala, Uppsala University; ZIN: Russia, Collection of Zoological Institute of Russian Academy of Science; ZIUH: China, Zhejiang University, Collection of Institute of Insect Sciences; ZMAN: Netherlands, Amsterdam, Universiteit van Amsterdam, Instituut voor Taxonomische Zoologie, Zoologisch Museum; ZMUC: Denmark, København [= Copenhagen], University of Copenhagen, Zoological Museum; ZMUK: Germany, Kiel, Universität Kiel, Zoologisches Museum; ZSM: Germany, München $\quad[=\quad$ Munich $], \quad$ Zoologische Staatssammlung.

\section{RESULTS AND DISCUSSION}

\section{Subfamily Acaenitinae Forster, 1869}

\section{Phalgea Cameron, 1905}

Phalgea maculata Pham \& van Achterberg, 2015

Phalgea maculata Pham \& van Achterberg, 2015. Zootaxa, 3947(1): 147. Holotype: ㅇ, Vietnam: Ha Noi, Khoang Xanh (RMNH). 
Distribution: Vietnam (Ha Noi) (Pham \& van Achterberg, 2015).

Phalgea melaptera Wang, 1989

Phalgea melaptera Wang, 1989. Acta Ent. Sin., 32(2): 228. Holotype: + , China: Guangxi (IZCAS).

Distribution: Vietnam (Vinh Phuc), China (Pham \& van Achterberg, 2015).

Subfamily AGRIOTYPINAE Haliday, 1838

Agriotypus Curtis, 1832

Agriotypus chaoi Bennett, 2001

Agriotypus chaoi Bennett, 2001. Syst. Ent., 26(3): 351. Holotype: đ̂, Vietnam: Lao Cai. (ROME). 2001).

Distribution: Vietnam (Lao Cai) (Bennett,

Agriotypus masneri Bennett, 2001

Agriotypus masneri Bennett, 2001. Syst. Ent., 26(3): 353. Holotype: ô, Vietnam: Ha Tinh (IEBR, on long-term loan to AMNH). 2001).

Distribution: Vietnam (Ha Tinh) (Bennett,

Subfamily ANOMALONINAE Viereck, 1918

Tribe Gravenhorstiini Enderlein, 1912

Trichomma Wesmael, 1849

Trichomma cnaphalocrocis Uchida, 1928

Trichomma cnaphalocrocis Uchida, 1928. Jour. Faculty Agr. Hokkaido Imp. Univ. 21: 239: Holotype: + , ô, Formosa: Tainan (EIHU).

Distribution: Vietnam (Yen Bai), Japan, Taiwan, the Philippines (Pham, 1997).

Remarks: This species was recorded as a parasitoid of the rice leafroller Cnaphalocrocis medinalis (Crambidae) (Khuat \& Pham, 2007).

\section{Subfamily BANCHINAEWesmael, 1845}

Tribe Atrophini Seyrig, 1932

Leptobatopsis Ashmead, 1900

Leptobatopsis indica (Cameron, 1897)

Cryptus indicus Cameron, 1897. Mem. \& Proc. Manchester Lit. Phil. Soc. 41(4): 15. Holotype: $\widehat{\jmath}$, India: Khasi Hills in Assam (BMNH)
Distribution: Vietnam (Son La, Phu Tho, Hoa Binh, Nghe An, Lam Dong), Australia, China, India, Indonesia, Japan, Laos, Malaysia, Myanmar, Singapore, Sri Lanka,Taiwan,Thailand, the Philippines (Pham, 2002).

Tribe Glyptini Cushman \& Rohwer, 1920

Sachtlebenia Townes, 1963

Sachtlebenia dmitrii Broad, 2014

Sachtlebenia dmitrii Broad, 2014. 68. Proc. Russ. Ent. Soc. St Petersburg, 85(1): Holotype: , Vietnam: Lam Dong, Bidoup Nui Ba NP (RMNH).

Distribution: Vietnam (Lam Dong) (Broad, 2014).

(*) Sachtlebenia sexmaculata Townes, 1963

Sachtlebenia sexmaculata Townes, 1963. Beit. Ent., 13: 523. Holotype: q, China: Fukien(USU)

Distribution: Vietnam (Ha Giang, Ninh Binh), China, Taiwan.

\section{Subfamily CAMPOPLEGINAE Forster,} 1869

Casinaria Holmgren, 1859

Casinaria ajanta Maheshwary \& Gupta, 1977

Casinaria ajanta Maheshwary \& Gupta, 1977. Orient. Insects Monograph, 5: 135.Type: $\hat{\jmath}$, Indonesia: Kalimantan (USNM).

Distribution: Vietnam (Lang Son, Son La, Ha Noi, Hung Yen, Ha Nam, Nam Dinh, Ba Ria - Vung Tau), Indonesia, Malaysia(Pham, 1997).

Remarks: This species is known as a parasitoid of Parnara guttata (Hesperiidae).

Casinaria indubia (Morley, 1913)

Trophocamba indubia Morley, 1913. Fauna of British India, Hymenoptera 3(1): 475. Holotype: $q$, India: Bhim Tal (DZCU).

Distribution: Vietnam (Bac Thai = Bac Can + Thai Nguyen, Nghe An), India, Myanmar, Nepal (PPRI, 1976).

Casinaria pedunculata (Szépligeti, 1908) 
Campoplex pedunculata Szépligeti, 1908. Notes Leyden Mus. 29: 232. Holotype: ㅇ, Indonesia: Java (HMHN).

Distribution: Vietnam (Lang Son, Phu Tho, Ha Noi), China, India, Indonesia, Myanmar, Nepal, Russia, Taiwan (Pham, 1997).

Remarks: This species was recorded as a parasitoid of Parnara guttata (Hesperiidae) and Grammodes geometrica (Noctuidae) (Pham \& Dang, 2009).

Charops Holmgren, 1859

Charops bicolor (Szépligeti, 1906)

Agrypon bicolor Szépligeti, 1906. Ann. Mus. Natl. Hungarici, 4: 124. Holotype: + , Ceylon: Colombo (HNHM).

Distribution: Vietnam (Lang Son, Phu Tho, Ha Noi, Hung Yen, Thai Binh, Quang Ngai, Phu Yen, Ba Ria-Vung Tau, Tien Giang), Australia, Bangladesh, China, India, Indonesia, Japan, Korea, Malaysia, Pakistan, Russia, Sri Lanka, Taiwan, Thailand (Pham, 1997).

Remarks: This species is known as a parasitoid of Mythimna separata,Naranga aenescens, Spodoptera litura (Noctuidae), Parnara guttata, Pelopidas mathias (Hesperiidae) (Khuat \& Pham, 2007).

(*) Charops brachypterus (Cameron, 1897)

Anomalon brachypterumCameron, 1897. Mem. \& Proc. Manchester Lit. Phil. Soc., 41(4): 25. Holotype: $\hat{\alpha}$, Sri Lanka: Trincomalee (OUMNH).

Distribution: Vietnam (Phu Tho, Ha Noi), China, India, Indonesia, Korea, Pakistan, Sri Lanka, Taiwan.

Remarks: It is the first record of this species from Vietnam.

Eriborus Forster, 1869

Eriborus argenteopilosus (Cameron, 1907)

Zaporus argenteopilosus Cameron, 1907. Jour. Bombay Nat. Hist. Soc. 17(3): 590. Holotype:, , India: Deesa in Bombay (BMNH).

Distribution: Vietnam (Yen Bai, Ha Noi, Nghe An), India, Indonesia, Myanmar (PPRI, 1976).
Eriborus ryukyuensis Momoi, 1970

Eriborus ryukyuensis Momoi, 1970. Pac. Insects, 12(2): 388. Holotype: †, Japan: Ryukyu Islands (EIHU).

Distribution: Vietnam (Ha Noi), Japan (Pham, 1997).

Remarks: This species was recorded as a parasitoid of Cnaphalocrocis medinalis (Crambidae).

Eriborus sinicus (Holmgren, 1868)

Limneria sinica Holmgren, 1868. Zoologi, 6: 412. Holotype: + , China (NHRS).

Distribution: Vietnam (Ha Noi), China, Japan, Taiwan, the Philippines, USA (Vu, 1986).

Eriborus vulgaris (Morley, 1913)

Dioctes vulgaris Morley, 1913. Fauna of British India, Hymenoptera, 3(1): 472. Holotype: + , India: Chapra in Bihar (BMNH).

Distribution: Vietnam (Ha Noi, Hung Yen $=$ Hai Duong + Hung Yen (now), Nam Ha = Nam Dinh + Ha Nam (now), China, India, Japan, Korea, Pakistan, Seychelles, Sri Lanka, Taiwan (Pham, 1997).

Remarks: This species was reared from Cnaphalocrocis medinalis (Crambidae).

\section{Subfamily CREMASTINAE Forster, 1869}

Temelucha Forster, 1869

Temelucha biguttula (Matsumura, 1910)

Ophionellus biguttulus Matsumura 1910.Extra Rpt. Agr. Exp. St. Aomori, 2: 67. Holotype: + , Japan: Aomori (EIHU).

Distribution: Vietnam $($ Vinh $\mathrm{Phu}=\mathrm{Vinh}$ Phuc + Phu Tho (now)), Bangladesh, China, Indonesia, Japan, Korea, Taiwan, USA (PPRI, 1976).

Temelucha philippinensis Ashmead, 1904

Temelucha philippinensis Ashmead, 1904. Jour. N.Y. Ent. Soc. 12: 18. Holotype: ㅇ, Philippinensis: Bacoor on Luzon (USNM).

Distribution: Vietnam (Thai Nguyen, Ha Noi, Bac Ninh, Hai Hung = Hai Duong + Hung Yen (now), Quang Ninh, Thai Binh, Nam Ha = 
Nam Dinh + Ha Nam (now), Phu Yen, Ba RiaVung Tau, Phu Yen), Bangladesh, China, India, Malaysia, Taiwan, Thailand, the Philippines (Pham, 1997).

Remarks: This species is known as a parasitoid of Cnaphalocrocis medinalis, Scirpophaga incertulas, Chilo suppressalis, Chilotrea auricilia (Crambidae), Naranga aenescens (Noctuidae), Parnara guttata (Hesperiidae), and Brachmia sp. (Gelechiidae) (Khuat \& Pham, 2007).

Temelucha stangli (Ashmead, 1904)

Leptopygus stangli Ashmead, 1904. Jour. N.Y. Ent. Soc. 12: 18. Holotype: Philippinensis: Bacoor on Luzon (USNM).

Distribution: Vietnam (Ha Noi, Tien Giang), Bangladesh, China, India, Malaysia, Philippines, Thailand (Vu, 1986).

Trathala Cameron, 1899

Trathala flavoorbitalis (Cameron, 1907)

Tarytia flavo-orbitalis Cameron, 1907. Jour. Bombay Nat. Hist. Soc.17: 589. Holotype: q, India: Deesa in Bombay (BMNH).

Distribution: Vietnam (Ha Noi, Thai Binh, Nam Dinh, Ha Nam, Tien Giang), Bangladesh, Canada, China, Fiji, Guam, India, Indonesia, Japan, Japan, Korea, Madagascar, Malaysia, Mariana Islands, Marshall Islands, Myanmar, Palau, Russia, Réunion, Singapore, Sri Lanka, Taiwan, The Philippines, Truk Islands, USA (Pham, 1997).

Remarks: This species is known as a parasitoid of Cnaphalocrocis medinalis, Scirpophaga incertulas, and Lamprosema indica (Crambidae) (Khuat \& Pham, 2007).

\section{Subfamily CRYPTINAE Kirby, 1837}

Tribe Cryptini Kirby, 1837

Subtribe Agrothereutina Townes, 1970

AmauromorphaAshmead, 1905

Amauromorpha accepta (Tosquinet, 1913)

Ischnoceros acceptus Tosquinet 1903. Mém. Soc. Ent. Belgique, 10: 67. Holotype: ㅇ, Indonesia: Java (IRSNB).

Distribution: Vietnam (Lang Son, Vinh
Phuc, Ha Noi, Hai Hung = Hai Duong + Hung Yen (now), Ha Nam, Nam Dinh, Thai Binh, Quang Ngai, Phu Yen, Can Tho, Tien Giang), China, India, Indonesia, Malaysia, Sri Lanka, Taiwan, the Philippines (Pham, 1997).

Remarks: This species was recorded as a parasitoid of Scirpophaga incertulas, Chilo suppressalis (Crambidae), Sesamia inferrens (Noctuidae) (Khuat \& Pham, 2007).

Subtribe Baryceratina Townes, 1961

BuysmaniaCheesman, 1941

Buysmania oxymora (Tosquinet, 1903)

Cryptus oxymorus Tosquinet, 1903. Mém. Soc. Ent. Belgique, 10: 214. Holotype: $q$, Indonesia: Java (IRSNB).

Distribution: Vietnam, China, Indonesia, Malaysia, Taiwan, Thailand, the Philippines (Pham, 2002).

Chlorocryptus Cameron, 1903

(*) Chlorocryptus purpuratus (Smith, 1852)

Cryptus purpuratus Smith, 1852. Trans. Ent. Soc. London (n.s.) 2: 33. Holotype: q, China: Ningpo-foo (BMNH).

Distribution: Vietnam (Ha Noi, Hoa Binh), China, India, Indonesia, Malaysia, Nepal.

Subtribe Gabuniina Townes, 1970

Schreineria Schreiner, 1905

Schreineria ceresia (Uchida, 1940)

Pseudotorbda ceresia Uchida, 1940. Ins. Matsumurana, 14: 119. Holotype: + , China: Kiangsu-Shanghai (CMNH).

Distribution: Vietnam, China (Pham, 2002).

Subtribe Goryphina Townes, 1970

Allophatnus Cameron, 1905

Allophatnus fulvitergus (Tosquinet, 1903)

Cryptus fulvitergus Tosquinet, 1903. Mém. Soc. Ent. Belgique, 10: 199. Holotype: ふ઼, Indonesia: Sumatra, Pangerang-Pissang (MCCI).

Distribution: Vietnam (Vinh Phuc, Ha Noi, Hoa Binh), China, India, Indonesia, Japan, Taiwan (Pham, 2002).

Goryphus Holmgren, 1868 
Goryphus basilaris Holmgren, 1868

Goryphus basilaris Holmgren, 1868. Kongliga Svenska Fregatten Eugenies Resa omkring jorden. Zoologi, 6: 398. Holotype: + , China (NHRS).

Distribution: Vietnam (Lang Son, Lao Cai, Lai Chau, Ha Noi, Hai Duong, Hung Yen, Thai Binh, Ha Nam, Nam Dinh), China, India, Indonesia, Japan, Malaysia, Myanmar, Taiwan (Pham, 1997).

Remarks: This species is known as a parasitoid of Cnaphalocrocis medinalis, Scirpophaga incertulas, Chilo suppressalis (Crambidae), Sesamia inferens (Noctuidae), Parnara guttata, Pelopidas mathias (Hesperiidae), and Brachmia sp. (Gelechiidae) (Khuat \& Pham, 2007).

SubtribeIschnina Holmgren, 1890

Etha Cameron, 1903

Etha tuberculata (Uchida, 1932)

Cryptus tuberculatus Uchida, 1932. Jour. Faculty Agr. Hokkaido Imp. Univ. 33: 168. Lectotype: 9 , Taiwan: Taihorin (DEI).

Distribution: Vietnam, China, Taiwan (Pham, 2002).

Subtribe Mesostenina Ashmead, 1900

Gotra Cameron, 1902

Gotra marginata (Brulle, 1846)

Mesostenus marginatus Brulle, 1846. In Lepeletier: Historie naturelle des insectes. Hyménopteres, 4: 224. Lectotype: 9 , India: Bengal (MNHN).

Distribution: Vietnam, China, India, Korea, Taiwan, the Philippines (Pham, 2002).

Gotra octocincta (Ashmead, 1906)

Mesostenus octocincta Ashmead, 1906. Proc. U.S. Natl. Mus. 30: 175. Holotype: + , China (USNM).

Distribution: Vietnam, China, Japan, Korea, Taiwan (Pham, 2002).

Subtribe Osprynchotina Viereck, 1918

Nematopodius Gravenhorst, 1829

Subgenus Diapetus Cameron, 1902 1905)

Nematopodius (Diapetus) luteus (Cameron,

Earrana lutea Cameron, 1905. Spolia Zeylanica, 3: 120. Holotype: $\widehat{\partial}$, Sri Lanka: Kandy (BMNH).

Synonym: Parca ocularia Morley (PPRI, 1976).

Distribution: Vietnam (Bac Thai $=$ Bac Kan + Thai Nguyen (now), Yen Bai, Ha Noi, Thai Binh, Ninh Binh), China, Indonesia, Sri Lanka, the Philippines (PPRI, 1976).

Tribe Phygadeuontini Forster, 1869

Subtribe Acrolytina Townes, 1970

Diatora Forster, 1869

Diatora prodeniae Ashmead, 1904

Diatora prodeniae Ashmead, 1904. Proc. U.S. Natl. Mus. 28: 141. Holotype: +, Philippines: Manila on Luzon (USNM).

Distribution: Vietnam (Ha Noi, Hoa Binh, Ha Nam), China, Malaysia, Taiwan, the Philippines(Khuat \& Pham, 2007).

Remarks: This species was recorded as aparasitoid of Cnaphalocrocis medinalis (Crambidae) (Khuat \& Pham, 2007).

Subtribe Bathytrichina Townes, 1970

Bathythrix Forster, 1869

Bathythrix kuwanae Viereck, 1912.

Bathythrix kuwanae Viereck, 1912. Proc. U.S. Natl. Mus. 43: 584. Holotype: ㅇ, Japan: Tokyo (USNM).

Distribution: Vietnam (Thai Nguyen, Ha Noi), China, Japan, Korea, Taiwan (Pham, 1997).

\section{Subfamily CTENOPELMATINAE Forster, 1869}

Tribe Chrionotini Uchida 1957

Chrionota Uchida, 1957

(*) Chrionota townesi Uchida, 1957

Chrionota townesi Uchida, 1957. Ins. Mats., 21: 42. Holotype: 9 , Formosa: Funkiko (EIHU).

Distribution: Vietnam (Son La), China, Taiwan. 
Remarks: It is the first record of this species from Vietnam.

Tribe Perilissini Thomson, 1883

Lathrolestes Foerster, 1869

(*) Lathrolestes roerichi Reshchikov, 2011

Lathrolestes roerichi Reshchikov, 2011. Zootaxa, 2743: 50. Holotype: +, Nepal, Kathmandu, Godavari (CNC).

Distribution: Vietnam (Son La), Nepal (Reshchikov, 2011).

Remarks: It is the first time this species has been recorded from Vietnam.

Metopheltes Uchida, 1932.

Metopheltes clypeoarmatus Reshchikov \&van Achterberg, 2014

Metopheltesclypeoarmatus Reshchikov \& van Achterberg, 2014. Bio. Data Jour., 2: 8. Holotype: 9 , Vietnam: Ninh Binh, Cuc Phuong NP (RMNH).

Distribution: Vietnam (Ninh Binh) (Reshchikov \& van Achterberg, 2014).

Neurogenia Roman, 1910

Neurogenia auco Reshchikov \& van Achterberg, 2014

Neurogenia auco Reshchikov \& van Achterberg, 2014. Tdschr. v. Ent., 157: 125. Holotype: 9 , Vietnam: Dak Lak, Chu Yang Sin NP (RMNH).

Distribution: Vietnam (Dak Lak) (Reshchikov et al., 2014).

Neurogenia gorochovi (Kasparyan, 2008)

Incurvarioni gorochovi Kasparyan, 2008. Zoosyst. Rossica: 85. Holotype: $\hat{\jmath}$, Vietnam: Gia Lai, Buon-Loi, (ZIN). 2008)

Distribution: Vietnam (Gia Lai) (Kasparyan,

Subfamily DIPLAZONTINAE Viereck, 1918

Diplazon Nees, 1819

Diplazon laetatorius (Fabricius, 1781)

Ichneumon laetatorius Fabricius, 1781. Species insectorum. Tom. I. Hamburgii et Kilonii: 424. Holotype: (sex not given), Germany (lost).
Distribution: Vietnam (Ha Noi, Hoa Binh), Afghanistan, Albania, Argentina, Australia, Austria, Azerbaijan, Azores, Belarus, Belgium, Brazil, Bulgaria, Burundi, Canada, Canary Islands, Chile, China, Cook Islands, Costa Rica, Croatia, Cyprus, Czech Republic, Czechoslovakia, Democratic Republic of Congo, Egypt, Estonia, Ethiopia, Fiji, Finland, France, Germany, Greece, Guam, Guatemala, Hungary, Iceland, India, Indonesia, Iran, Ireland, Isle of Man, Israel, Italy, Japan, Korea, Latvia, Lesotho, Libya, Lithuania, Luxembourg, Madagascar, Madeira Islands, Mariana Islands, Mexico, Midway Islands, Moldova, Mongolia, New Caledonia, New Zealand, Norway, Pakistan, Papua New Guinea Peru, Poland, Portugal, Romania, Russia, Rwanda, Réunion, Senegal, Society Islands, South Africa, Spain,St. Helena, Sudan, Sweden, Switzerland, Taiwan, Tajikistan,the Netherlands, the Philippines, Tonga, Tunisia, Turkey, Turkmenistan, U.S.A, Uganda, Ukrain, United Kingdom, Uruguay, Uzbekistan, Vanuatu, Western Samoa, Yugoslavia, Zambia, Zimbabwe (Pham, 2002).

\section{Subfamily ICHNEUMONINAE Latreille, 1802}

Tribe Compsophorini Heinrich, 1967

Compsophorus Saussure, 1892

Compsophorus caeruleus (Cameron, 1901)

Compsophorus caeruleus caeruleus (Cameron, 1901)

Charitojoppa caeruleus (Cameron, 1901). Ann.\& Mag. Nat. Hist. (7) 7: 384. Lectotype: 9 , India: Khasi Hills in Assam (OUMNH).

Distribution: Vietnam (Ninh Binh, Dong Nai), China, Indonesia, Myanmar (Riedel, 2011).

Compsophorus gracilis (Heinrich, 1975)

Charitojoppa gracilis Heinrich, 1975. Ann. Zool. 32:483. Holotype: +, Burma: Toungoo (ZMHB)

Distribution: Vietnam (Dong Nai), Myanmar (Riedel, 2011).

Compsophorus vietnamensis (Riedel, 2011) 
Habrojoppa vietnamensis Riedel, 2011. Linz. Biol. Beitr., 43 (2): 1558. Holotype: $\widehat{\partial}$, Vietnam: Viet Try (RMNH). 2011).

Distribution: Vietnam (Phu Tho) (Riedel,

Tribe Goedartiini Townes, 1961

Maraces femoralis (Cameron, 1907)

Felargia femoralis Cameron, 1907. Tijdschr.v. Ent. 50: 83. Holotype: + , India: Sikkim (BMNH).

Distribution: Vietnam (Phu Tho), India, Laos, Myanmar (Riedel, 2011).

Maraces flavobalteata Cameron, 1902

Maraces flavobalteata vietnamensis Riedel, 2011

Maraces flavobalteata vietnamensis Riedel, 2011. Linz. Biol. Beitr., 43(2): 1559. Holotype:

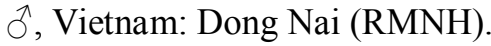
2011)

Distribution: Vietnam (Dong Nai) (Riedel,

Maraces rufothoracica Riedel 2011

Maraces rufothoracica Riedel 2011. Linz. Biol. Beitr., 43(2): 1560. Holotype: đ̂, Vietnam: Viet Try (RMNH). 2011).

Distribution: Vietnam (Phu Tho) (Riedel,

Tribe Heresiarchini Ashmead, 1900

Aglaojoppa Cameron, 1901

Aglaojoppa rothneyi Cameron, 1902

Aglaojoppa rothneyi Cameron, 1902. Ann. \& Mag. Nat. Hist., (7) 9: 145. Lectotype: + , India: Khasi Hills in Assam (OUMNH).

Distribution: Vietnam (Lao Cai), India, Myanmar (Riedel, 2013).

Aglaojoppa rufofemorata Cameron, 1903

Aglaojoppa rufofemorata Cameron, 1903. Ann. \& Mag. Nat. Hist., (7) 12: 363. Holotype: , India: Khasi Hills in Assam (OUMNH).

Distribution: Vietnam (Dak Lak), China, India, Indonesia, Myanmar, Nepal (Riedel, 2013)

\section{Amblyjoppa Cameron, 1902 \\ Amblyjoppa sinensis (Heinrich, 1931)}

Protichneumon sinensis Heinrich, 1931. Ztschr. f. Angew. Ent., 18: 399. Holotype: ô, : China: Kwangtung (MZPW).

Distribution: Vietnam (Ninh Binh), China, Myanmar (Riedel, 2013).

Amblyjoppa sulcata (Cameron, 1906)

Glyptojoppa sulcata Cameron, 1906. Entomologist, 39: 229. Holotype: $\hat{\sigma}$, Burma: Salween Valley in Tenasserim division (BMNH).

Distribution: Vietnam (Phu Tho), Myanmar (Riedel, 2013).

Atanyjoppa Cameron, 1901

Atanyjoppa lineata Heinrich, 1968

Atanyjoppa lineata Heinrich, 1968. Ent. Tidskrift. 89: 220. Holotype: ${ }^{\text {, Burma: Mt. }}$ Victoria (MZPW)

Distribution: Vietnam (Dak Lak), India, Myanmar (Riedel, 2013).

Atanyjoppa rufipes Heinrich 1968

Atanyjoppa rufipes Heinrich 1968. Ent. Tidskrift. 89: 221. Holotype: 9 , Burma : Maymyo (MZPW).

Distribution: Vietnam (Dong Nai), Myanmar (Riedel, 2013).

Atanyjoppa rufomaculata Cameron, 1901

Atanyjoppa

rufomaculatarufomaculataCameron, 1901. Proc. Zool. Soc. London, (2): 38. Holotype: ㅇ, India: Khasi Hills in Assam (OUMNH).

Distribution: Vietnam (Lao Cai, Phu Tho, Ninh Binh, Thua Thien-Hue), China, India, Myanmar (Riedel, 2013).

Coelichneumon Thomson, 1893

Subgenus Coelichneumon Thomson, 1893

Coelichneumon

(Coelichneumon)

birmanicus Heinrich, 1966

Coelichneumon birmanicus Heinrich, 1966. Ent. Tidskrift. 87: 208. Holotype: ${ }^{\circ}$, Burma : Mt. Victoria (ZSM).

Distribution: Vietnam (Dak Lak), Myanmar (Riedel, 2013).

Coelichneumon (Coelichneumon) pieli Uchida, 1937 
Coelichneumon pieli Uchida, 1937. Ins.Matsumurana, 11: 86. Holotype: 우, China : Kuling (EIHU).

Distribution: Vietnam (Vinh Phuc), China (Riedel, 2013).

Coelichneumon (Coelichneumon) taihorinus Uchida, 1932

Coelichneumon taihorinus Uchida, 1932. Jour.Fculty Agr. Hokkaido Univ.,33: 148. Lectotype: + , Formosa: Taihorin (DEI).

Distribution: Vietnam (Thua Thien-Hue), Bhutan, China, India, Indonesia, Myanmar, Nepal Taiwan (Riedel, 2013). 1934

Subgenus Intermedichneumon Heinrich,

Coelichneumon ocellus (Tosquinet, 1903)

Ichneumon ocellus Tosquinet, 1903. Mém. Soc. Ent. Belgique, 10: 319. Holotype: + , Indonesia: Java (IRSNB).

Distribution: Vietnam (Dong Nai), China, India, Indonesia, Japan, Myanmar, Nepal, Singapore, Taiwan, Thailand, the Philippines (Pham, 2002).

Tribe Ichneumonini Latreille, 1802

Stenichneumon Thomson, 1893

(*) Stenichneumon appropinquans (Cameron, 1897)

Ichneumon appropinquans Cameron, 1897. Mem. \& Proc. Manchester Lit. Phil. Soc. 41(4): 5. Holotype: +, India: Mussoorie in U.P. (OUMNH).

Distribution: Vietnam (Son La, Phu Tho, Vinh Phuc, Ha Noi, Ninh Binh, Kon Tum), China, India, Korea, Nepal, Sri Lanka.

Ulesta Cameron, 1903

Ulesta agitata (Matsumura \& Uchida, 1926)

Chasmias agitata Matsumura \& Uchida, 1926. Jour. Faculty Agr. Hokkaido Imp. Univ. 18: 72. Holotype:우 Japan: Hokkaido(EIHU).

Distribution: Vietnam, China, Japan, Korea (Pham, 2002).

Tribe Ischnojoppini Heinrich, 1938

Ischnojoppa Kriechbaumer, 1898
Ischnojoppa luteator (Fabricius, 1798)

Ichneumon luteator Fabricius, 1798. Supp. Ent. Syst.: 222. Lectotype: + , Germany: Halle in Saxony (ZMUC).

Synonym: Atanyjoppa akonis Matsumura (PPRI, 1976).

Distribution: Vietnam (Lang Son, Lao Cai, Bac Thai $=$ Bac Can + Thai Nguyen (now), Yen Bai, Hoa Binh), Australia, Bangladesh, China, Taiwan, India, Indonesia, Japan, Kenya, Korea, Malaysia, Myanmar, Papua New Guinea, Senegal, Singapore, South Africa, Sri Lanka Taiwan, the Philippines, Uganda (PPRI,1976).

Remarks: This species was reared from Parnara guttata, Pelopidas mathias (Hesperiidae) (Khuat \& Pham, 2007).

Tribe Listrodromini Forster, 1869

Listrodromus Wesmael, 1845

Listrodromus crassipes (Cameron, 1907)

Sogna crassipesCameron, 1907. Tijdschr.v. Ent. 50: 92. Holotype: 9 , India: Sikkim (BMNH).

Distribution: Vietnam (Dak Lak), China, India, Indonesia, Malaysia, Taiwan (Riedel, 2011).

Tribe Phaeogenini Forster, 1869

Auberteterus Diller, 1981 1929)

Auberteterus alternecoloratus (Cushman,

Centeterus alternecoloratus Cushman, 1929. Proc. Hawaii. Ent. Soc. 7: 243. Holotype: 9 , China: Foochow in Fukien (USNM).

Distribution: Vietnam (Yen Bai, Vinh Phu = Phu Tho + Vinh Phuc (now)), China, France, India, Russia, Taiwan (Pham, 1997).

Remarks: This species has been known as parasitoid of Chilo suppressalis (Crambidae) (Khuat \& Pham, 2007).

Tribe Platylabini Berthoumieu, 1904

Apaeleticus tonkinensis Riedel, 2011

Apaeleticus tonkinensis Riedel, 2011. Linz. Biol. Beitr., 43 (2): 1561. Holotype: ㅇ, Vietnam: Hoang Lien NP (RMNH). 

2011).

Distribution: Vietnam (Lao Cai) (Riedel, Asthenolabus Heinrich 1951

Asthenolabus major Heinrich, 1974

Asthenolabus major Heinrich 1974. Ann. Zool., 32:170. Holotype: 옹 Burma: Kambaiti (NHRS).

Distribution: Vietnam (Lao Cai), Myanmar (Riedel, 2011).

Asthenolabus vietnamensis Riedel, 2011

Asthenolabus vietnamensis Riedel, 2011. Linz. Biol. Beitr., 43(2): 1562. Holotype: $\widehat{0}$, Vietnam: Hoang Lien NP (RMNH). 2011).

Distribution: Vietnam (Lao Cai) (Riedel,

Clypeolabus Heinrich, 1974

Cratolabus formosanus (Uchida, 1932)

Platylabus formosanus Uchida, 1932. Jour. FacultyAgr. Hokkaido Univ. 33: 162. Lectotype: $\widehat{\jmath}$, Formosa: Taihorin (DEI).

Distribution: Vietnam (Lao Cai, Vinh Phuc), China, India, Laos, Myanmar, Taiwan (Riedel, 2011).

Cyclolabus Heinrich, 1936

Cyclolabus basirufus Heinrich, 1974

Cyclolabus basirufus Heinrich, 1974. Ann. Zool., 32: 144. Holotype: ㅇ, Burma: Kambaiti (NHRS).

Distribution: Vietnam (Lao Cai), Myanmar (Riedel, 2011).

Notoplatylabus Heinrich, 1936

Notoplatylabus birmanicus Heinrich, 1974

Notoplatylabus birmanicus Heinrich, 1974.Ann. Zool., 32: 177. Holotype: ㅇ, Burma: Kambaiti (NHRS).

Distribution: Vietnam (Dak Lak), China, India, Indonesia, Laos Malaysia, Taiwan (Riedel, 2011).

Pachyjoppa Cameron, 1901

Pachyjoppa tibialis Cameron, 1901

Pachyjoppa tibialis Cameron, 1901. Ann. \& Mag. Nat. Hist., 7 (7): 375. Holotype: + , India: Khasi Hills in Assam (OUMNH).
Distribution: Vietnam (Lao Cai), India, Myanmar (Riedel, 2011).

Pagarenes Cameron, 1903

Pagarenes erythropus Cameron, 1903

Pagarenes erythropus Cameron, 1903. Ztschr. System. Hymen. Dipt., 3: 184. Holotype: + , India: Sikkim (BMNH).

Distribution: Vietnam (Lao Cai), India; Laos; Myanmar (Riedel, 2011).

Paraplatylabus Riedel, 2011

Paraplatylabus vietnamensis Riedel, 2011

Paraplatylabus vietnamensis Riedel, 2011. Linz. Biol. Beitr. 43(2): 1570. Holotype: 9 , Vietnam: Lam Dong (RMNH).

Distribution: Vietnam (Lam Dong) (Riedel, 2011).

Platylabus Wesmael, 1845

Platylabus basicinctus Heinrich, 1974

Platylabus basicinctus Heinrich, 1974. Ann. Zool., 32: 165. Holotype: §̂, Burma: Mt. Victoria (MZPW).

Distribution: Vietnam (Lao Cai), Myanmar (Riedel, 2011).

Platylabus flagellator Riedel, 2011

Platylabus flagellator Riedel, 2011. Linz. Biol. Beitr., 43(2): 1565. Holotype: + , Vietnam: Tam Dao NP (RMNH).

Distribution: Vietnam (Vinh Phuc) (Riedel, 2011).

Platylabus fuscinerva (Cameron, 1901)

Lamprojoppa fuscinerva Cameron 1901. Ann. \& Mag. Nat. Hist., 12: 574. Holotype: + , India: Khasi Hills in Assam (BMNH).

Distribution: Vietnam (Lao Cai), India, Nepal (Riedel, 2011).

Pristicerops Heinrich, 1961

Pristicerops principalis Heinrich, 1974

Pristicerops principalis Heinrich 1974. Ann. Zool., 32: 165. Holotype: ㅇ, Burma: Mt. Victoria (MZPW).

Distribution: Vietnam (Lao Cai), Myanmar (Riedel, 2011). 
Pristicerops rufithorax Riedel, 2011

Pristicerops rufithorax Riedel, 2011. Linz. Biol. Beitr., 43(2): 1568. Holotype: ${ }^{\Uparrow}$, Vietnam: Hoang Lien NP (RMNH). 2011)

Distribution: Vietnam (Lao Cai) (Riedel,

\section{Subfamily MESOCHORINAE Forster, 1869}

Stictopisthus Thomson, 1886

Stictopisthus chinensis (Uchida, 1942)

Mesochoruschinensis Uchida, 1942. Ins. Mats., 16: 130. Holotype: + , China: Hangzhou (EIHU).

Distribution: Vietnam, China (Pham, 2002).

\section{Subfamily METOPIINAE Forster, 1869}

Acerataspis Uchida, 1934

(*) Acerataspis clavata (Uchida, 1934)

Cerataspis clavata Uchida, 1934. Trans. Sapporo Nat. Hist. Soc. 13: 277. Holotype: $\widehat{\partial}$, Japan (KUEC).

Distribution: Vietnam (Vinh Phuc, Ha Noi, Phu Tho, Hoa Binh), China, Japan, Korea, Russia.

Remarks: It is the first record of this species from Vietnam.

Colpotrochia Holmgren, 1856

Colpotrochia pilosa (Cameron, 1909)

Inoresa pilosa Cameron, 1909. Jour. Bombay Nat. Hist. Soc. 19: 724. Holotype: $\widehat{\partial}$, India: Simla (BMNH).

Distribution: Vietnam (Ninh Binh, Nghe An, Ha Tinh, Quang Nam, Dong Nai), China, India, Japan, Korea, Pakistan, Taiwan, the Philippines (Pham, 2007).

Exochus Gravenhorst, 1829

Exochus scutellatus (Morley, 1913)

Xanthexochus scutellatus Morley, 1913. Fauna of British India, Hymenoptera, 3: 293. Holotype: $\hat{O}$, India: Chandkhira, Sylhet in Assam (BMNH).

Distribution: Vietnam (Thai Nguyen, Ha Noi), Bangladesh, China, India (Pham, 2007).

Hypsicera Latreille 1829
Hypsicera lita (Chiu, 1962)

Hypsicera lita Chiu, 1962. Bull. Taiwan Agri. Res. Inst. 20: 26. Holotype: $\widehat{\delta}$, Taiwan: Wushe (TARI).

Distribution: Vietnam (Ha Giang, Ha Noi, Hoa Binh, Ninh Binh), China, Taiwan (Chiu, 1962; Pham, 2007).

Metopius Panzer, 1806

Subgenus Ceratopius Clément, 1927 1930

Metopius (Ceratopius) baibarensis Uchida,

Metopius baibarensis Uchida, 1930. Jour. Faculty Agr. Hokkaido Imp. Univ. 25: 250: Holotype: $\delta^{\lambda}$, Taiwan: Baibara, Naihonpo (EIHU).

Distribution: Vietnam (Thai Nguyen), China, Taiwan (Pham, 2007). 1785)

Metopius (Ceratopius) citratus (Geoffroy,

Ichneumon citratus Geoffroy, 1785. In Fourcroy, A.F. de. Entomologia parisiensis, sive catalogus insectorum quae in agro Parisiensi reperiuntur. Pars secunda. Via et Aedibus Serpentineis, Paris: 403. Holotype: ㅇ (lost).

Synonym: Metopius (Ceratopius) dissectorius Panzer (Pham, 2004).

Distribution: Vietnam (Hoa Binh, Quang Nam), Austria, Belgium, China, Croatia, Czechoslovakia, Finland, France, Germany, Greece, Hungary, India, Ireland, Israel, Japan, Korea, Latvia, Nepal, Netherlands, Norway, Poland, Romania, Russia, Spain, Switzerland, Taiwan, Turkey, Ukraine, United Kingdom, Yugoslavia (Pham, 2004). 1941

Metopius (Ceratopius) metallicus Michener,

Metopius metallicus Michener, 1941. PanPacific Ent. 17: 7. Holotype: đ, China: Wong Sa Shui in south Kiangsi (CAS)

Distribution: Vietnam (Hoa Binh, Ninh Binh), China, Taiwan (Pham, 2007).

Subgenus Metopius Panzer 1806

Metopius (Metopius) rufus browni Ashmead, 1905 
Metopius browni Ashmead, 1905. Proc. U.S. Natl. Mus. 29: 117. Holotype: $\widehat{\partial}$, Philippines: Manila on Luzon (USNM).

Metopius rufus Cameron, 1905. Ztschr. System. Hymen. Dipt. 5: 281. Holotype: ㅇ, India: Mt. Abu in Rajasthan (BMNH).

Distribution: Vietnam (Thai Nguyen, Ha Noi, Hoa Binh, Ninh Binh, Nghe An, Thua Thien-Hue, Quang Nam), China, India, Indonesia, Japan, Korea, Mongolia, Myanmar, Taiwan, Thailand, the Philippines (Pham, 2004).

Remarks: This species was recorded as a parasitoid of Parnara guttata (Hesperiidae), and Mythimna separata (Noctuidae) (Khuat \& Pham, 2007).

Triclistus Foerster, 1869

Triclistus aitkeni (Cameron, 1897)

Exochus aitkini Cameron, 1897. Mem. \& Proc. Manchester Lit. Phil. Soc. 41(4): 31. Holotype: $\hat{\jmath}$, India: Bengal (OUMNH).

Distribution: Vietnam (Ninh Binh), China, India, Japan, Korea, Russia, Réunion, Taiwan, the Philippines (Pham\& Khuat, 2007).

\section{Subfamily OPHIONINAE Shuckard, 1840}

Enicospilus Stephens, 1835

Enicospilus aciculatus (Taschenberg, 1875)

Ophion aciculatus Taschenberg, 1875. Ztschr.f. d.Ges. Naturw., 46: 434. Lectotype: Indonesia: Java (MLUH).

Distribution: Vietnam (Quang Ninh), China, India, Indonesia, Japan, Myanmar, Solomon Islands, Sri Lanka, Taiwan, the Philippines, Vanuatu (Gauld \& Mitchell, 1981).

Enicospilus ashbyi Ashmead, 1904

Enicospilus ashbyi Ashmead 1904. Jour. New York Ent. Soc., 12: 17: Holotype: $\widehat{\partial}$, Philippines (USNM).

Distribution: Vietnam (Hoa Binh), China, India, Indonesia, Malaysia, Papua New Guinea, Singapore, Taiwan, the Philippines (Gauld \& Mitchell, 1981).

Enicospilus bharatensis Nikam, 1980

Enicospilus indicus Rao \& Nikam, 1969:
143. Holotype: + , India (MUC). [Junior primary homonym of Enicospilus indica Rao \& Grover, 1960].

Enicospilus bharatensis Nikam, 1980. Orient. Ins., 14(2): 182. Replacement name for indicus Rao \& Nikam, 1969.

Distribution: Vietnam (North), China, India, Indonesia, New Caledonia, Papua New Guinea, Sri Lanka, the Philippines, Vanuatu (Gauld \& Mitchell, 1981).

Enicospilus dolosus (Tosquinet, 1896)

Ophion dolosus Tosquinet, 1896. Mem. Soc. Ent. Belgique. 5: 389. Holotype: + , Egypt (ZMHB).

Distribution: Vietnam (Quang Nam), Angola, Australia, Bangladesh, Brunei, Central African Republic, China, Democratic Republic of Congo, Egypt, Ethiopia, Fiji, Gabon, Ghana, Guinea, India, Indonesia, Ivory Coast, Kenya, Madagascar, Malaysia, Mauritania, Mozambique, New Caledonia, Nigeria, Papua New Guinea, Réunion, Senegal, Society Islands, Solomon Islands, South Africa, Sri Lanka, Taiwan, Tanzania, the Philippines, Uganda, Vanuatu, Western Samoa (Gauld \& Mitchell, 1981).

Enicospilus flavicaput (Morley, 1912)

Enicospilus xanthocephalus Cameron, 1907. Ann. Mag. Natur. Hist., (7)19: 178. Holotype: , , Burma (BMNH) [Junior primary homonym of Enicospilus xanthocephalus Cameron, 1905).

Henicospilus flavicaput Morley, 1912. British Museum: 45 [Replacement name for xanthocephalus Cameron, 1907).

Distribution: Vietnam (Hoa Binh?), Brunei, China, India, Indonesia, Japan, Malaysia, Myanmar, Nepal, Sri Lanka, the Philippines (Gauld \& Mitchell, 1981). 1921)

Enicospilus grammospilus (Enderlein,

Dicamptus grammospilus Enderlein, 1921. Stett. Ent. Zeit., 82: 17. Holotype: ô, Indonesia: Sumatra (MZPW).

Distribution: Vietnam (Tuyen Quang), Brunei, China, India, Indonesia, Malaysia, 
Taiwan, the Philippines (Gauld \& Mitchell, 1981).

(*) Enicospilus hirayamai Uchida, 1955

Allocamptus orientalis Uchida, 1928. Jour. Faculty Agr. Hokkaido Imp. Univ. 21: 230. Holotype: $\hat{\jmath}$, $q$, Formosa: Baibara; Pingtung. Ryukyu Is.: Okinawa (EIHU). Name preoccupied in Enicospilus by Morley, 1913.

Enicospilus hirayamai Uchida, 1955. Jour. Faculty Agr. Hokkaido Imp. Univ., 50: 120.

Distribution: Vietnam (Phu Tho, Vinh Phuc, Ha Noi), China, Japan, Taiwan.

Enicospilus kanshirensis (Uchida, 1928)

Henicospilus kanshirensis Uchida, 1928. Journal of the Faculty of Agriculture, Hokkaido University. 21: 226. Holotype: ふ̄, Taiwan (EIHU).

Distribution: Vietnam (Lai Chau, Hoa Binh), Brunei, China, India, Indonesia, Myanmar, Nepal, Taiwan, the Philippines (Gauld \& Mitchell, 1981).

Enicospilus lineolatus (Roman, 1913)

Henicospilus lineolatus Roman, 1913. Arkiv för Zool. 8(15): 30. Holotype: $\widehat{\jmath}$, Philippines (NHRS).

Synonym: Enicospilus striatus Cameron, 1899 (PPRI, 1976).

Distribution: Vietnam (Yen Bai, Vinh Phu = Vinh Phuc + Phu Tho (now), Hoa Binh, Ha Noi, $\mathrm{Nam} \mathrm{Ha}=\mathrm{Nam}$ Dinh $+\mathrm{Ha}$ Nam (now)), Australia, Brunei, China, India, Indonesia, Japan, Korea, Malaysia, Nepal, New Caledonia, Papua New Guinea, Singapore, Solomon Islands, Sri Lanka, Taiwan, Tajikistan, the Philippines (PPRI, 1976).

Remarks: This species was reared from the corn borer Ostrinia furnacalis (Crambidae) (Khuat \& Pham, 2007).

Enicospilus maai Chiu, 1954

Enicospilus maai Chiu, 1954. Bull. Taiwan Agr. Res. Inst., 13: 22. Holotype: +, Taiwan (TARI).

Distribution: Vietnam (Quang Nam), China, India, Malaysia, Taiwan (Gauld \& Mitchell, 1981).
Enicospilus maritus (Roman, 1913)

Henicospilus maritus Roman, 1913. Arkiv för Zool., 8(15): 29. Holotype: ふ઼, Philippines (NHRS).

Distribution: Vietnam (Lang Son?), Brunei, China, Indonesia, Malaysia the Philippines (Gauld \& Mitchell, 1981).

(*) Enicospilus melanocarpus Cameron, 1905

Enicospilus melanocarpus Cameron, 1905. Spolia Zeylanica, 3: 122. Holotype: 9 , Sri Lanka: Kandy (BMNH).

Distribution: Vietnam (Son La, Phu Tho, Hoa Binh, Ha Noi, Vinh Phuc, Bac Giang), Australia, Brunei, China, India, Japan, Malaysia, Myanmar, Nepal, New Caledonia, Pakistan, Papua New Guinea, the Philippines, Singapore, Society Islands, Solomon Islands, Sri Lanka, Taiwan, Vanuatu, Western Samoa.

Enicospilus plicatus (Brullé, 1846)

Ophion plicatus Brulle 1846. Hist. Natur. Ins.: 145. Holotype: + , Java (DEZC).

Distribution: Vietnam (Hoa Binh), Brunei, China, India, Indonesia, Malaysia, Papua New Guinea, Singapore, Taiwan, Thailand, the Philippines (Gauld \& Mitchell, 1981).

Enicospilus pseudantennatus Gauld, 1977

Enicospilus pseudantennatus Gauld, 1977. Australian Journal of Zoology (Supplementary Series), 49: 92. Holotype: +, Australia (ANIC).

Distribution: Vietnam (South), Australia, China, India, Indonesia, Myanmar, Nepal, Norfolk Island, Papua New Guinea, Sri Lanka, Taiwan, the Philippines (Gauld \& Mitchell, 1981).

Enicospilus pudibundae (Uchida 1928)

Henicospilus pudibundae Uchida 1928. Jour. Faculty Agr., Hokkaido Univ., 21: 219. Lectotype: $\hat{\sigma}$, Japan (EIHU).

Distribution: Vietnam (Hoa Binh), Brunei, China, India, Japan, Korea, Laos, Sri Lanka (Gauld \& Mitchell, 1981).

Enicospilus rogus Gauld \& Mitchell, 1981

Enicospilus rogus Gauld \& Mitchell, 1981. Commonwealth Inst. Ent.: 351. Holotype: $q$, 
Brunei (BMNH).

Distribution: Vietnam, Brunei, India (Gauld \& Mitchell, 1981).

Enicospilus shinkanus (Uchida, 1928)

Henicospilus shinkanus Uchida, 1928. Jour. Faculty Agr., Hokkaido Univ., 21: 217. Holotype: +, Taiwan (EIHU).

Distribution: Vietnam (Ho Chi Minh city), Chagos Archipelago, China, Fiji, India, Japan, Korea, Malaysia, New Caledonia, Palau, Papua New Guinea, Solomon Islands, Taiwan, the Philippines, Vanuatu, Western Samoa (Gauld \& Mitchell, 1981).

Enicospilus signativentris (Tosquinet, 1903)

Ophion signativentris Tosquinet, 1903. Mem. Soc. Ent. Belgique. 10: 37. Lectotype: ㅇ, Indonesia: Java (IRSNB).

Distribution: Vietnam (Hoa Binh), Bhutan, China, India, Indonesia, Japan, Malaysia, Sri Lanka, Taiwan, the Philippines (Gauld \& Mitchell, 1981).

Enicospilus stenophleps Cushman 1937

Enicospilus stenophleps Cushman 1937. Arb. über Morph.u. Taxonom. Ent., 4: 309. Holotype: $q$, Taiwan (DEI).

Distribution: Vietnam (Ho Chi Minh city), China, India, Sri Lanka, Taiwan (Gauld \& Mitchell, 1981).

Enicospilus vestigator (Smith, 1858)

Ophion vestigator Smith, 1858. Jour. Proc. Linn. Soc. London, Zool., 2:122. Holotype: $\curvearrowright$, Malaysia (OUMNH).

Distribution: Vietnam, Brunei, China, India, Indonesia, Malaysia, Palau, Papua New Guinea, Sri Lanka, Taiwan (Gauld \& Mitchell, 1981).

\section{Subfamily PIMPLINAE Wesmael, 1845}

Tribe Ephialtini Hellen, 1915

Acrodactyla Haliday, 1838

Acrodactyla elongata Pham, Broad, Matsumoto \& Böhme, 2012

Acrodactyla elongata Pham, Broad, Matsumoto \& Böhme, 2012. Zootaxa, 3207: 41. Holotype: + , Vietnam: Lao Cai, Hoang Lien NP (RMNH).
Distribution: Vietnam (Lao Cai) (Pham et al., 2012a).

Acrodactyla lachryma Pham, Broad, Matsumoto \& Böhme, 2012

Acrodactyla lachryma Pham, Broad, Matsumoto \& Böhme, 2012. Zootaxa, 3207: 45. Holotype: + , Vietnam: Kontum, Chu Mom Ray NP (RMNH).

Distribution: Vietnam (Lao Cai) (Pham et al., 2012a).

Acrodactyla maiphuquyi Pham, Broad, Matsumoto \& Böhme, 2012

Acrodactyla maiphuquyi Pham, Broad, Matsumoto \& Böhme, 2012. Zootaxa, 3207: 48.Holotype: $q$, Vietnam: Lao Cai, Hoang Lien NP (RMNH).

Distribution:Vietnam (Lao Cai) (Pham et al., 2012a).

Acrodactyla phuthoensis Pham, Broad, Matsumoto \& Böhme, 2012

Acrodactyla phuthoensis Pham, Broad, Matsumoto \& Böhme, 2012. Zootaxa, 3207: 49.Holotype: 9 , Vietnam: Phu Tho, Xuan Son NP (RMNH).

Distribution: Vietnam (Lao Cai) (Pham et al., 2012a).

Acrodactyla shawi Pham, Broad, Matsumoto \& Böhme, 2012

Acrodactyla shawi Pham, Broad, Matsumoto \& Böhme, 2012. Zootaxa, 3207: 51. Holotype: $q$, Vietnam: Lao Cai, Sapa (OMNH).

Distribution: Vietnam (Lao Cai) (Pham et al., 2012a).

Acrodactyla tami Pham, Broad, Matsumoto \& Böhme, 2012

Acrodactyla tami Pham, Broad, Matsumoto \& Böhme, 2012. Zootaxa, 3207: 52. Holotype: q, Vietnam: Lao Cai, Hoang Lien NP (RMNH).

Distribution:Vietnam (Lao Cai) (Pham et al., 2012a).

Acropimpla Townes, 1960

Acropimpla hapaliae (Rao, 1953)

Philopsyche hapaliae Rao, 1953. Indian 
Forest Rec. Ent., 8: 168. Holotype: ${ }^{\text {, India: }}$ Mallipatna in Coorg, Mysore (IFRI).

Distribution: Vietnam (Son La, Phu Tho, Hoa Binh, Nghe An, Dong Nai), China, India, Myanmar (Pham et al., 2011d). 2011

Acropimpla lampei Pham, Broad \&Wägele,

Acropimpla lampei Pham, Broad, Wägele, 2011. Zootaxa, 2921: 6. Holotype: ㅇ (IEBR), Dak Lak, Ea So NR.

Distribution: Vietnam (Dak Lak) (Pham et al.,2011d).

Acropimpla leucostoma (Cameron, 1907)

Charitopimpla leucostoma Cameron, 1907. Tijdschr. Ent., 50: 97. Holotype: q, India: Sikkim (BMNH).

Distribution:Vietnam (Vinh Phuc, Lai Chau, Phu Tho, Hoa Binh, Ninh Binh, Ha Tinh, Thua Thien-Hue, Kon Tum, Dak Lak, Dong Nai), China, India, Indonesia, Japan, Laos, Myanmar, Sri Lanka (Pham, 2013).

Acropimpla mucronis Pham, Broad \&Wägele, 2011

Acropimpla mucronis Pham, Broad, Wägele, 2011. Zootaxa, 2921: 7. Holotype: $q$ (RMNH), Dong Nai, Cat Tien NP.

Distribution: Vietnam (Dong Nai) (Pham et al., 2011d).

Acropimpla phongdienensis Pham, Broad \& Wägele, 2011

Acropimpla phongdienensis Pham, Broad, Wägele, 2011. Zootaxa, 2921: 9. Holotype: 오, Thua Thien-Hue, Phong Dien NR (RMNH).

Distribution: Vietnam (Thua Thien-Hue) (Pham et al., 2011d). 2010

Acropimpla taishunensis Liu, He\& Chen,

Acropimpla taishunensis Liu, He \& Chen, 2010. Zootaxa, 2394: 36. Holotype: + , China: Zhejiang (ZJUH).

Distribution: Vietnam (Nghe An), China (Pham et al., 2011d).

Brachyzapus Gauld \& Dubois, 2006

Brachyzapus carinatus Pham, Broad,
Matsumoto \& Wägele, 2012

Brachyzapus carinatus Pham, Broad, Matsumoto \& Wägele, 2012. J. Nat. Hist. 46(27-28): 1640. Holotype: +, Vietnam: Lao Cai, $\mathrm{Sa} \mathrm{Pa}(\mathrm{OMNH})$.

Distribution: Vietnam (Lao Cai) (Pham et al., 2012b).

Brachyzapus convexus Pham, Broad, Matsumoto \& Wägele, 2012

Brachyzapus convexus Pham, Broad, Matsumoto \& Wägele, 2012. J. Nat. Hist. 46(27-28): 1643. Holotype: q, Vietnam: Lao Cai, Hoang Lien NP, Fansipan Mt (RMNH).

Distribution: Vietnam (Lao Cai) (Pham et al., 2012b).

Brachyzapus duboisi Pham, Broad, Matsumoto \& Wägele, 2012

Brachyzapus duboisi Pham, Broad, Matsumoto \& Wägele, 2012. J. Nat. Hist. 46(27-28): 1646. Holotype: +, Vietnam: Lao Cai, Hoang Lien NP, Fansipan Mt (RMNH).

Distribution: Vietnam (Lao Cai) (Pham et al., 2012b).

Brachyzapus fansipanensisPham, Broad, Matsumoto \& Wägele, 2012

Brachyzapus fansipanensis Pham, Broad, Matsumoto \& Wägele, 2012. J. Nat. Hist. 46(27-28): 1649. Holotype: q, Vietnam: Lao Cai, Hoang Lien NP, Fansipan Mt (RMNH).

Distribution: Vietnam (Lao Cai) (Pham et al., 2012b).

Brachyzapus hoanglienensisPham, Broad, Matsumoto \& Wägele, 2012

Brachyzapus hoanglienensis Pham, Broad, Matsumoto \& Wägele, 2012. J. Nat. Hist. 46(27-28): 1652. Holotype: + , Vietnam: Lao Cai, Hoang Lien NP (RMNH).

Distribution: Vietnam (Lao Cai) (Pham et al., 2012b).

Brachyzapus politus Pham, Broad, Matsumoto \&Wägele, 2012

Brachyzapus politus Pham, Broad, Matsumoto \& Wägele, 2012. J. Nat. Hist. 46(27-28): 1654. Holotype: + , Vietnam: Lao 
Cai, Hoang Lien NP (RMNH).

Distribution: Vietnam (Lao Cai) (Pham et al., 2012b).

Camptotypus Kriechbaumer, 1889

Camptotypus arianus (Cameron, 1899)

Pimpla ariana Cameron, 1899. Mem. Proc. Manchester. Lit. Phil. Soc., 43(3): 157. Lectotype: + India: Khasi Hills (now in Meghalaya) (OUMNH).

Distribution: Vietnam (Son La, Hoa Binh, Thua Thien-Hue, Dong Nai), China, India, Indonesia, Laos, Myanmar, Taiwan (Gupta \& Tikar, 1976).

Camptotypus olynthius (Cameron, 1899)

Pimpla olynthia Cameron, 1899. Mem. Proc. Manchester Lit. Phil. Soc., 43(3): 156. Holotype: ㅇ, India: Khasi Hills (OUMNH).

Distribution: Vietnam (Vinh Phuc, Thua Thien-Hue), India, Indonesia, Myanmar, Thailand (Pham et al., 2012c).

Camptotypus testaceus (Cameron, 1907)

Erythropimpla testaceus Cameron, 1907. Tijdschr. Ent., 50: 98. Holotype: ${ }^{\circ}$, India: Sikkim (BMNH).

Distribution: Vietnam (Thanh Hoa, Ha Tinh, Dong Nai), India, Myanmar (Gupta \& Tikar, 1976). 2012

Camptotypus trui Pham, Broad \& Wägele,

Camptotypus trui Pham, Broad \& Wägele, 2012. Dtsch. Ent. Z., 59 (1): 134. Holotype: 우, Vietnam: Kon Tum, Chu Mom Ray NP (IEBR).

Distribution: Vietnam (Kon Tum, Nghe An) (Pham et al., 2012c).

Chablisea Gauld \& Dubois, 2006

Chablisea condimenta Pham, Broad, Matsomoto \& Wägele, 2011

Chablisea condimenta Pham, Broad, Matsomoto \& Wägele, 2011. Biologia 66/6: 1134. Holotype: + , Vietnam: Lao Cai, Hoang Lien NP (RMNH).

Distribution: Vietnam (Lao Cai) (Pham et al., 2011b).
Chablisea khuatdanglongi Pham, Broad, Matsomoto \& Wägele, 2011

Chablisea khuatdanglongi Pham, Broad, Matsomoto \& Wägele, 2011. Biologia 66/6: 1136. Holotype: + , Vietnam: Ha Noi, Thach That, Tan Xa (IEBR).

Distribution: Vietnam (Ha Noi, Hoa Binh, Ninh Binh, Kon Tum, Dak Lak) (Pham et al.,2011b).

Dolichomitus Smith, 1877

Dolichomitus lamiPham, Broad \&Zwakhals, 2012

Dolichomitus lami Pham, Broad \& Zwakhals, 2012. Zootaxa, 3519: 78. Holotype: , Vietnam: Lao Cai (Hoang Lien NP) (IEBR).

Distribution: Vietnam (Lao Cai) (Pham et al., 2012d).

Dolichomitus melanomerus (Vollenhovenn,1878)

Ephialtes melanomerus Vollenhoven, 1878. Tijdschr. Ent. 21: 48. Holotype: क, Java (RMNH)

Distribution: Vietnam (Vinh Phuc, Hoa Binh), India, Indonesia, Japan, Russia, Sri Lanka, Taiwan, the Philippines (Pham et al., 2012d)

Flavopimpla Betrem, 1932 2013

Flavopimpla lanugoPham, Broad \&Wägele,

Flavopimpla lanugoPham, Broad \&Wägele, 2013. Biologia 68/4: 721. Holotype: o, Vietnam: Phu Tho (BMNH).

Distribution: Vietnam (Phu Tho) (Pham et al., 2013c).

Flavopimpla latiannulata (Cameron, 1907)

Ephialtes latiannulatus Cameron, 1907. Tijdschr. Ent., 50: 96. Holotype: , India: Sikkim (BMNH).

Distribution: Vietnam (Ha Noi, Dong Nai), China, India, Sri Lanka (Pham et al., 2011e).

Flavopimpla vinhcuuensis Pham, Broad\& Wägele, 2013

Flavopimpla vinhcuuensis Pham, Broad \& Wägele, 2013. Biologia 68/4: 723. Holotype: 
q, Vietnam: Dong Nai (IEBR).

Distribution: Vietnam (Dong Nai) (Pham et al., 2013c).

Gregopimpla Momoi, 1965 1899)

Gregopimpla himalayensis (Cameron,

Pimpla himalayensis Cameron, 1899. Mem. \& Proc. Manchester Lit. Phil. Soc., 43 (3): 178. Holotype: + , India: Khasil Hills in Assam (OUMNH)

Distribution: Vietnam (Lao Cai), China, India, Japan, Korea (Pham et al., 2011e).

Leptopimpla Townes, 1961

Leptopimpla longiventris (Cameron, 1908)

Ephialtes longiventris Cameron, 1908. Ztschr. System. Hymen. Dept., 8: 37. Holotype: ㅇ,

India: Sikkim (BMNH).

Distribution: Vietnam (Hoa Binh, Thua Thien-Hue, Quang Nam), Nepal, India, Myanmar, Malaysia, Indonesia, Taiwan (Gupta \& Tikar, 1976).

Sericopimpla Kriechbaumer, 1895

Sericopimpla sagrae (Vollenhoven, 1879)

Pimpla sagrae Vollenhoven, 1879. Stettin. Ent. Ztg., 40: 149. Lectotype: 우 Indonesia: Java (RMNH).

Distribution: Vietnam (Thai Nguyen, Phu Tho, Ha Noi, Hoa Binh, Nghe An, Ha Tinh, Thua Thien-Hue, Dak Lak, Dong Nai), China, India, Indonesia, Japan, Korea, Malaysia, Philippines, Sri Lanca, Taiwan(Pham et al., 2011e).

Zaglyptus Foerster, 1869

Zaglyptus glaber Gupta, 1961

Zaglyptus glaber Gupta, 1961. Indian J. Ent., 22(1960): 252.

Distribution: Vietnam (Thai Nguyen, Phu Tho, Vinh Phuc, Ninh Thuan), China, India, Singapore (Pham et al., 2013d). 2013

Zaglyptus guptai Pham, Broad \& Wägele,

Zaglyptus guptai Pham, Broad \& Wägele,
2013. Zootaxa, 3702(5): 486. Holotype: $q$, Vietnam: Dak Lak, Chu Yang Sin NP (RMNH).

Distribution:Vietnam (Dak Lak) (Pham et al., 2013d).

\section{Zaglyptus nigrolineatus Gupta, 1961}

Zaglyptus nigrolineatus Gupta, 1961. Indian J. Ent., 22 (1960): 255. Holotype: + India: Cherangode (CNC).

Distribution: Vietnam (Dak Lak), India (Pham et al., 2013d).

\section{Zatypota Forster, 1869}

Zatypota albicoxa (Walker, 1874)

Glypta albicoxa Walker, 1874.Cistula Ent., 1:304. Holotype: + , $(\mathrm{BMNH})$

Distribution: Vietnam (Lao Cai), Austria, Belarus, Bulgaria, China, Czech Republic, Czechoslovakia, Denmark, Finland, France, Germany, India, Japan, Moldova, Netherlands, Poland, Romania, Russia, Sweden, Ukraine, United Kingdom (Tanasuka et al., 2012).

Tribe Pimplini Wesmael, 1845

Augerella Gupta, 1962

Augerella achterbergi Pham, Broad \& Lampe, 2011

Augerella achterbergi Pham, Broad \& Lampe, 2011. Zootaxa, 2745: 68. Holotype: + , Vietnam: Dak Lak, Chu Yang Sin NP (RMNH).

Distribution: Vietnam (Dak Lak) (Pham et al.,2011a).

Augerella brevicauda (Cushman, 1933)

Theronia brevicauda Cushman, 1933. Ins. Matsumurana 8: 49. Holotype: $\$$, Taihorin (DEI).

Theronia (Augerella) orientalis Gupta, 1962. Pac. Ins. Mon. 4: 87. Holotype: ㅇ, Philippines: Mt. Canlaon, $1100 \mathrm{~m}$, Negros Oriental (USU).Synonimized with $A$. brevicauda by Pham et al., 2013b.

Distribution: Vietnam (Vinh Phuc, Ha Noi, Nghe An, Ninh Thuan, Dong Nai), China, India, Indonesia, Malaysia, the Philippines (Pham et al., 2013b).

Augerella vriesi Pham, Broad \& Lampe, 
Augerella vriesi Pham, Broad \& Lampe, 2011. Zootaxa, 2745: 68. Holotype: $ᄋ$, Vietnam: Dak Lak, Chu Yang Sin NP (RMNH).

Distribution: Vietnam (Dak Lak) (Pham et al., 2011a).

\section{Echthromorpha Holmgren, 1868} 1787)

Echthromorpha agrestoria (Swederus,

Ichneumon agrestorius Swederus, 1787. Kongliga Svenska Vetenskapsakademiens Handlingar. 8: 279 . Holotype: + , Tahiti (BMNH).

Echthromorpha agrestoria notulatoria (Fabricius, 1804)

Cryptus notulatorius Fabricius, 1804. Systema piezatorum: 77. Holotype: + , India: Tranquebar in Madras (ZMUK).

Distribution: Vietnam (Ha Giang, Son La, Thai Nguyen, Vinh Phuc, Ha Noi, Phu Tho, Hoa Binh, Ninh Binh, Thanh Hoa, Nghe An, Ha Tinh, Quang Binh, Thua Thien-Hue, Da Nang, Kon Tum, Dong Nai), Australia, Bangladesh, Cameroon, China, Democratic Republic of Congo, Egypt, Ethiopia, Fiji, Guam, Guinea, India, Indonesia, Japan, Kenya, Laos, Liberia, Madagascar, Malaysia, Mauritius, Mozambique, Myanmar, New Caledonia, Palau, Papua New Guinea, Réunion, Senegal, Sierra Leone, Singapores, Society Islands, South Africa, Sri Lanca, Sudan, Tanzania, Thailand, the Philippines, Togo, Uganda, U.S.A, Vanuatu, Zimbabwe (Pham, 2013).

Remarks: This species was recorded as a parasitoid of Anomis flava (Erebidae) (Khuat \& Pham, 2007).

Epitheronia Gupta, 1962

Epitheronia vinhanensis Pham, Broad \& Wägele, 2013

Epitheronia vinhanensis Pham, Broad \& Wägele, 2013. Journal of Natural History: 1509. Holotype: + , Vietnam: Dong Nai (IEBR).

Distribution: Vietnam (Dong Nai) (Pham et al., 2013b).

Itoplectis Foerster, 1869

Itoplectis naranyae (Ashmead, 1906)
Nesopimpla naranyae Ashmead, 1906. Proc. U. S. Natl. Mus. 30: 180. Holotype: ㅇ, Japan: Hokkaido, Sapporo (USNM).

Distribution: Vietnam (Ha Noi, Ninh Binh), China, Japan, Korea, Russia, Taiwan, the Philippines (Vu, 1986; Bui, 1990).

Remarks: This species was recorded as aparasitoid oflepidopteran pests: Cnaphalocrocis medinalis (Crambidae), Parnara guttata, Pelopidas mathias (Hesperiidae), Naranga aenescens (Noctuidae), and Brachmia sp. (Gelechiidae) (Vu, 1986; Bui, 1990).

Lissopimpla Kriechbaumer, 1889

Lissopimpla basalis (Vollenhoven, 1879)

Pimpla basalis Vollenhoven, 1879. Stettin Ent. Ztg. 40(4-6): 148. Holotype: ${ }^{\top}$, Indonesia: Sumatra (RMNH).

Distribution: Vietnam (Ha Noi, Hoa Binh, Ninh Binh, Nghe An, Ha Tinh, Thua ThienHue, Quang Nam, Dak Lak), Indonesia, the Philippines (Pham, 2013).

Nomosphecia Gupta, 1962

Nomosphecia carinicurvata Pham, Broad \& Wägele, 2013

Nomosphecia carinicurvata Pham, Broad \& Wägele, 2013. Journal of Natural History: 1513. Holotype: + , Vietnam: Dong Nai (RMNH).

Distribution: Vietnam (Dong Nai) (Pham et al., 2013b).

Nomosphecia scutellata (Gupta, 1962)

Theronia (Nomosphecia) scutellata Gupta, 1962. Pac. Ins. Mon. 4: 81.

Theronia (Nomosphecia) scutellata scutellata Gupta, 1962. Pac. Ins. Mon. 4: 82. Holotype: $\circ$, Singapore (USNM).

Distribution: Vietnam (Ninh Binh, Dak Lak, Dong Nai), Malaysia, Singapore, the Philippines (Pham et al., 2013b).

Nomosphecia zebroides (Krieger, 1906)

Theronia zebroides Krieger, 1906. Ztschr. Syst. Hymen. Dipt. 6: 236. Holotype: + , Indonesia: Sumatra (ZMHB).

Distribution: Vietnam (Son La, Phu Tho, 
Vinh Phuc, Nghe An, Quang Binh, Quang Tri, Dak Lak, Dong Nai), India, Indonesia, Japan, Malaysia, Taiwan (Pham et al., 2013b).

Parema Gupta, 1962

Parema nigrobalteata (Cameron, 1899)

Theronia nigrobalteata Cameron, 1899. Mem. \& Proc. Manchester Lit. Phil. Soc. 43(3): 153. Holotype: 9 , India: Khasi Hills, Assam (OUMNH).

Distribution: Vietnam (Tuyen Quang, Phu Tho, Vinh Phuc, Bac Giang, Hoa Binh, Ninh Binh, Nghe An, Ha Tinh, Thua Thien-Hue, Quang Nam, Kon Tum, Dak Lak, Ninh Thuan, Dong Nai), India, Indonesia, Malaysia, Myanmar, Singapore, Thailand, the Philippines (Gupta, 1962).

Pimpla Fabricius, 1804

Pimpla aethiops Curtis, 1828

Pimpla aethiops Curtis, 1828. British Ent. 5: 214. Lectotype: + , Great Britain: reared from Laelia coenosa (Lepidoptera: Erebidae: Lymantriinae) (MVMA).

Distribution: Vietnam (Ha Noi), Austria, Bulgaria, China, Czechoslovakia, France, Germany, Hungary,Iceland, Italy, Japan, Korea, Russia, Spain, Taiwan (Bui, 1990).

Remarks: This species is known as parasitoid of Parnara guttata, Pelopidas mathias (Hesperiidae), Brachmiasp. (Gelechiidae), Naranga aenescens (Noctuidae), and Cnaphalocrocis medinalis (Crambidae) (Khuat \& Pham, 2007).

Pimpla bilineata (Cameron, 1900)

Habropimpla bilineata Cameron, 1900. Mem. \& Proc. Manchester Lit. Phil. Soc., 44(15): 97. Holotype: $\widehat{\jmath}$, India: Meghalaya: Khasi Hill (BMNH).

Distribution: Vietnam (Lao Cai), China, India, Myanmar, Nepal (Pham et al., 2013a).

Pimpla cameronii Dalla Torre, 1901

Pimpla vidua Cameron, 1899. Mem. \& Proc. Manchester Lit. Phil. Soc. 43(3): 180. Name preoccupied by Pimpla vidua Walsh, 1873 (= Tromatobia ovivora (Boheman, 1821)). Holotype: $\widehat{\jmath}$, India: Meghalaya: Khasi Hills

\section{(OUMNH).}

Pimpla cameronii Dalla Torre, 1901. Catalogus Hymenopterorum, 3.

Distribution: Vietnam (Lao Cai, Hoa Binh, Son La), India, Indonesia, Myanmar, Nepal, Taiwan (Pham et al., 2013a).

Pimpla carinifrons Cameron, 1899

Pimpla carinifrons Cameron, 1899. Mem. Proc. Manchester Lit. Phil. Soc. 43(3): 172. Lectotype: + , India: Meghalaya, Khasi Hills (BMNH).

Distribution: Vietnam (Lao Cai, South Vietnam (imprecise locality)), China, India, Laos, Myanmar, Nepal, Taiwan (Gupta \& Saxena, 1987).

Pimpla chuyangsinensis Pham, Broad, Dang \& Böhme, 2013

Pimpla chuyangsinensis Pham, Broad, Dang \& Böhme, 2013. Org. Divers. Evol. 13(3): 401. Holotype: đ̃, Vietnam: Dak Lak, Chu Yang Sin NP (RMNH).

Distribution: Vietnam (Dak Lak) (Pham et al., 2013a).

\section{Pimpla ereba Cameron, 1899}

Pimpla ereba Cameron, 1899. Mem. Proc. Manchester Lit. Phil. Soc. 43(3): 172. Holotype: , India: Meghalaya, Khasi Hills (OUMNH).

Distribution: Vietnam (Lao Cai), China, India, Myanmar (Pham et al., 2013a).

Pimpla flavipalpis Cameron, 1899

Pimpla flavipalpis Cameron, 1899. Mem. \& Proc. Manchester Lit. Phil. Soc., 43(3): 174. Lectotype: + , India: Meghalaya: Khasi Hill (BMNH).

Distribution: Vietnam (Lao Cai, Kon Tum), China, India, Myanmar, Nepal, Taiwan (Pham et al. 2013a).

\section{Pimpla laothoe Cameron, 1897}

Pimpla laothoe Cameron, 1897. Mem. \& Proc. Manchester Lit. Phil. Soc., 41(4): 22.

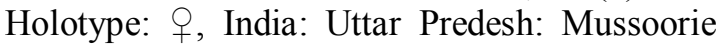
(OUMNH).

Distribution: Vietnam (Lao Cai, Vinh Phuc, Hoa Binh, Ha Noi), China, India, Indonesia, 
Myanmar, Nepal, Pakistan, Sri Lanka, Taiwan (Pham et al., 2013a).

Remarks: This species was reared from Parnara guttata (Hesperiidae).

Pimpla lexuanhuei Pham, Broad, Dang \&Böhme, 2013

Pimpla lexuanhuei Pham, Broad, Dang \&Böhme, 2013. Org. Divers.Evol. 13(3): 405. Holotype: 9 , Vietnam: Phu Tho (IEBR).

Distribution: Vietnam (Phu Tho) (Pham et al., 2013a).

Pimpla nipponica Uchida, 1928

Pimpla nipponica Uchida, 1928. Jour. Faculty Agr. Hokkaido Imp. Univ. 25: 45. Lectype: 9 , Japan: Hokkaido: Sapporo (EIHU).

Distribution: Vietnam (Son La, Ha Noi), China, Finland, India, Japan, Korea, Russia (Bui, 1990).

Remarks: This species is known as a parasitoid of Parnara guttata, Pelopidas mathias (Hesperiidae), Brachmia sp. (Gelechiidae), Naranga aenescens (Noctuidae), and Cnaphalocrocis medinalis (Crambidae) (Khuat \& Pham, 2007).

Theronia Holmgren, 1859

Theronia clathrata Krieger, 1899

Theronia clathrata Krieger, 1899. Naturf. Gesell. Leipzig, Sitzber. 1897/98: 111. Holotype: O: India: Khasi Hills, Assam (ZMHB).

Distribution: Vietnam (Ha Tinh, Dak Lak), China, India, Indonesia, Malaysia, Sri Lanka, Taiwan, the Philippines (Gupta, 1962).

Theronia ferruginaterga Pham, Broad \& Wägele, 2013

Theronia ferruginaterga Pham, Broad \& Wägele, 2013. Journal of Natural History, 47(23-24): 1525. Holotype: 9 , Vietnam: Kon Tum, Chu Mom Ray NP (RMNH).

Distribution: Vietnam (Hoa Binh, Thua Thien-Hue, Dak Lak) (Pham et al., 2013b).

Theronia longihastata Pham, Broad \& Wägele, 2013

Theronia longihastata Pham, Broad \&
Wägele, 2013. Journal ofNatural History, 47(23-24): 1528. Holotype: + , Vietnam: Dak Lak, Chu Yang Sin NP (RMNH).

Distribution:Vietnam (Dak Lak) (Pham et al, 2013b).

Theronia maskeliyae Cameron, 1905

Theronia maskeliyae Cameron, 1905. Spolia Zeylanica, 3: 133. Lectotype: ${ }^{+}$, Sri Lanka: Maskeliya (BMNH).

Distribution: Vietnam (Thai Nguyen, Vinh Phuc,Nghe An), China, India, Indonesia, Singapore, Sri Lanka, Thailand, the Philippines (Pham et al., 2013b).

Theronia tahuythinhi Pham, Broad \& Wägele, 2013

Theronia tahuythinhi Pham, Broad \& Wägele, 2013. Journal of Natural History, 47(23-24): 1532. Holotype: , Vietnam: Dak Lak, Chu Yang Sin NP (RMNH).

Distribution: Vietnam (Dak Lak) (Pham et al, 2013b).

Theronia zebra (Vollenhoven, 1879)

Pimpla zebra Vollenhoven, 1879. Stett. Ent. Ztg. 40: 147. Holotype: , Indonesia: Java, Ambarawa (RMNH).

Distribution: Vietnam (Cao Bang, Son La, Ha Noi, Vinh Phuc, Phu Tho, Hoa Binh, Nghe An, Ha Tinh, Thua Thien-Hue, Gia Lai), Brunei, China, India, Indonesia, Malaysia, Myanmar, Singapore, Sri Lanka, Taiwan, Thailand, the Philippines (Gupta, 1962).

Xanthopimpla Saussure, 1892

Xanthopimpla amplamaculosa Pham, Broad, Matsumoto \& Wägele, 2011

Xanthopimpla amplamaculosa Pham, Broad, Matsumoto \& Wägele, 2011. Zootaxa, 3056: 20. Holotype: + , Vietnam: Ninh Binh, Cuc Phuong NP (IEBR).

Distribution: Vietnam (Vinh Phuc, Ninh Binh) (Pham et al., 2011c).

Xanthopimpla alternans Krieger, 1914

Xanthopimpla alternans Krieger, 1914. Arch. f. Naturgesch., (A) 80(6): 31. Holotype: q, Taiwan: Chiayi (ZMHB). 
Distribution: Vietnam (Nghe An, Nha Trang, Gia Lai), China, India, Indonesia, Malaysia, Sri Lanka, Taiwan (Pham, 2013).

Xanthopimpla annulata Cushman, 1925

Xanthopimpla annulata Cushman, 1925. Ent. Mitt. 14: 47. Holotype: 9 , Taiwan: Kangkou [= Kankau], Hengchun (DEI).

Distribution:Vietnam (Phu Tho, Ha Noi, Hoa Binh), China, India, Taiwan, the Philippines (Pham et al., 2011c).

Xanthopimpla atriclunis Townes \& Chiu, 1970

Xanthopimpla atriclunis Townes \& Chiu, 1970. Mem. Amer. Ent. Inst., 14: 157. Holotype: $q$, the Philippines: Alcate, Victoria, Mindoro Oriental (USU).

Distribution: Vietnam (Thua Thien-Hue, Dak Lak, Dong Nai), the Philippines (Pham et al., 2011c).

Xanthopimpla brachycentra Krieger, 1914

Xanthopimpla brachycentra Krieger, 1914. Arch. f. Naturgesch., (A) 80(6): 40, 86. Lectotype: +,Taiwan: Chulotung [= Teraso] in Kaohsiung (ZMHB).

Distribution: Vietnam (Bac Can, Phu Tho, Ha Noi, Bac Giang, Nghe An, Ha Tinh), China, India, Taiwan (Pham et al., 2011c).

Xanthopimpla boehmei Pham, Broad, Matsumoto \& Wägele, 2011

Xanthopimpla boehmei Pham, Broad, Matsumoto \& Wägele, 2011. Zootaxa, 3056: 41. Holotype: $q$, Vietnam: Dak Lak, Chu Yang Sin NP (IEBR).

Distribution: Vietnam (Dak Lak) (Pham et al., 2011c).

Xanthopimpla brevicarina Wang, 1987

Xanthopimpla brevicarina Wang, 1987. Acta Ent. Sinica, 30(3): 328. Holotype: ㅇ, China: Ningming Co. Guangxi (IZCAS).

Distribution:Vietnam (Ninh Binh), China (Pham et al., 2011c).

Xanthopimpla calva Townes \& Chiu, 1970

Xanthopimpla calva Townes \& Chiu, 1970. Mem. Amer. Ent. Inst., 14: 240. Holotype: , the Philippines: Gapan, Nueva Ecija (USU).

Distribution: Vietnam (Nghe An, Dak Lak), India, Malaysia, Myanmar Philippines (Pham et al., 2011c).

Xanthopimpla chiuae Pham, Broad, Matsumoto \& Wägele, 2011

Xanthopimpla chiuae Pham, Broad, Matsumoto \& Wägele, 2011. Zootaxa, 3056: 22. Holotype: $q$, Vietnam: Dak Lak, Chu Yang Sin NP (RMNH).

Distribution: Vietnam (Dak Lak) (Pham et al., 2011c).

Xanthopimpla clivulus Townes \& Chiu, 1970

Xanthopimpla clivulus Townes \& Chiu, 1970. Mem. Amer. Ent. Inst., 14: 160. Holotype: $q$, Indonesia: Java (USU).

Distribution: Vietnam (Thai Nguyen, Vinh Phuc, Dak Lak), India, Indonesia, Malaysia, Singapore (Pham et al., 2011c).

Xanthopimpla conica Cushman, 1925

Xanthopimpla conica Cushman, 1925. Ent. Mitt., 14: 45. Holotype: + , Taiwan: Kangkou [= Kankau], Hengchun (DEI).

Distribution:Vietnam (Lai Chau, Phu Tho, Hoa Binh, Quang Nam, Dong Nai), China, India, Indonesia, Malaysia, Sri Lanka, Taiwan (Townes \& Chiu, 1970).

Xanthopimpla connexa Krieger, 1914

Xanthopimpla connexa Krieger, 1914. Arch. f. Naturgesch. (A) 80(6): 22. Holotype: 9 , the Philippines (ZMHB).

Distribution: Vietnam (Dong Nai, Ninh Thuan), India, the Philippines (Pham et al., 2011c). 1899)

Xanthopimpla curvimaculata (Cameron,

Pimpla curvimaculata Cameron, 1899. Mem. \& Proc. Manchester Lit. Phil. Soc. 43(3): 158. Holotype: ${ }^{\lambda}$, India: Khasi Hills in Assam (OUMNH).

Distribution: Vietnam (Phu Tho, Dak Lak, Dong Nai), India, Malaysia, Thailand (Pham et al., 2011c). 


\section{Xanthopimpla decurtata Krieger, 1914}

Xanthopimpla decurtata Krieger, 1914. Arch. f. Naturgesch., (A) 80(6): 39, 113. Lectotype: + , the Philippines: Atimonan on Luzon (ZMHB).

Distribution: Vietnam (Lai Chau, Vinh Phuc, Nghe An, Dong Nai), India, Malaysia, Taiwan, Thailand, the Philippines (Townes \& Chiu, 1970).

Xanthopimpla despinosa Krieger, 1914

Xanthopimpla despinosa Krieger, 1914. Arch. f. Naturgesch., (A) 80(6): 32. Holotype: $\widehat{\partial}$, Indonesia: Sumatra (MZPW).

Distribution: Vietnam (Lai Chau, Phu Tho, Nghe An, Dong Nai), India, Indonesia, Malaysia, Nepal, the Philippines (Townes \& Chiu, 1970).

Xanthopimpla elegans (Vollenhoven, 1879)

Pimpla elegans Vollenhoven, 1879. Stettin. Ent. Ztg. 40: 147. Holotype: + , Indonesia: Java (RMNH).

Distribution: Vietnam (Phu Tho, Ninh Binh, Nghe An, Ha Tinh, Quang Tri, Thua ThienHue, Kon Tum, Dak Lak, Lam Dong, Dong Nai), India, Indonesia, Laos, Malaysia, Myanmar, Nepal, Singapore, Sri Lanka, Taiwan, Thailand, the Philippines (Pham et al., 2011c).

\section{Xanthopimpla enderleini Krieger, 1914}

Xanthopimpla enderleini Krieger, 1914. Arch. f. Naturgesch., (A) 80(6): 35. Lectotype: , Indonesia: Sumatra (MZPW).

Distribution. Vietnam (Ha Noi, Hung Yen, Tien Giang, Binh Dinh, Phu Yen), China, India, Malaysia, Indonesia, the Philippines (Pham, 1997).

Remarks: This species was recorded as a parasitoid of lepidopteran pests on rice, including Cnaphalocrocis medinalis (Crambidae), Parnara guttata (Hesperiidae), and Sesamia inferens (Noctuidae) (Pham, 1997).

\section{Xanthopimpla fastigiata Krieger, 1914}

Xanthopimpla fastigiata Krieger, 1914. Arch. f. Naturgesch., (A) 80(6): 30. Holotype: $\widehat{\jmath}$, Indonesia: Sumatra, Sarik (ZMHB).

Distribution: Vietnam (Phu Tho, Ha Noi, Thua Thien-Hue, Dak Lak), India, Indonesia, Malaysia, Singapore, Thailand, the Philippines (Pham et al., 2011c).

Xanthopimpla flavafemora Pham, Broad, Matsumoto \& Wägele, 2011

Xanthopimpla flavafemora Pham, Broad, Matsumoto \& Wägele, 2011. Zootaxa, 3056: 26. Holotype: + , Vietnam: Nghe An, Pu Mat NP (IEBR).

Distribution: Vietnam (Nghe An) (Pham et al., 2011c).

Xanthopimpla flavapropodea Pham, Broad, Matsumoto \& Wägele, 2011

Xanthopimpla flavapropodea Pham, Broad, Matsumoto \& Wägele, 2011. Zootaxa, 3056: 27. Holotype: q, Vietnam: Bac Giang: Thanh Son (IEBR).

Distribution: Vietnam (Bac Can, Bac Giang, Nghe An) (Pham et al., 2011c).

Xanthopimpla flavolineata Cameron, 1907

Xanthopimpla flavolineata Cameron, 1907. Tijdschr. v. Ent., 50: 48. Holotype: , New Guinea: Merauke (ZMAN).

Distribution: Vietnam (Lai Chau, Son La, Thai Nguyen, Phu Tho, Vinh Phuc, Ha Noi, Bac Giang, Hung Yen, Hai Phong, Quang Ninh, Hoa Binh, Ninh Binh, Nghe An, Ha Tinh, Quang Tri, Quang Ngai, Phu Yen, Tien Giang, Ba RiaVung Tau), Australia, Bangladesh, China, India, Indonesia, Japan, Laos, Malaysia, Nepal, New Caledonia, Pakistan, Palau, Papua New Guinea, Sri Lanka, the Philippines, Vanuatu (Pham, 1997).

Remarks: This species has been reared from pupae of Cnaphalocrocis medinalis, Parnara guttata (Hesperiidae) and Naranga aenescens (Lepidoptera: Noctuidae) on rice and Lamprosema indica (Lepidoptera: Pyralidae) on soybean (Khuat \& Pham, 2007; Vu, 2007).

Xanthopimpla glaberrima Roman, 1913

Xanthopimpla glaberrima Roman, 1913. Arkiv för Zool. 8(15): 22. Holotype: $q$, the Philippines (NHRS). 
Distribution: Vietnam (Thai Nguyen, Phu Tho, Hoa Binh), India, Indonesia, Malaysia, Thailand, Taiwan, the Philippines (Pham et al., 2011c).

Xanthopimpla hienae Pham, Broad, Matsumoto \& Wägele, 2011

Xanthopimpla hienae Pham, Broad, Matsumoto \& Wägele, 2011. Zootaxa, 3056: 35. Holotype: $q$, Vietnam: Dak Lak, Ea So NR (IEBR).

Distribution: Vietnam (Dak Lak) (Pham et al., 2011c).

Xanthopimpla honorata (Cameron, 1899)

Pimpla honorata Cameron, 1899. Mem. \& Proc. Manchester Lit. Phil. Soc., 43(3): 170.

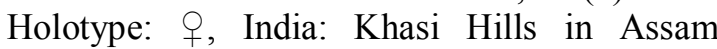
(OUMNH).

Distribution: Vietnam (Ha Giang, Vinh Phuc, Ha Noi, Hoa Binh, Ninh Binh, Nghe An, Quang Binh, Quang Tri, Thua Thien-Hue), China, India, Indonesia, Laos, Malaysia, Myanmar, Nepal, Singapore, Taiwan, Thailand, the Philippines (Townes \& Chiu, 1970).

Xanthopimpla jacobsoni Krieger, 1914

Xanthopimpla jacobsoni Krieger, 1914. Arch. f. Naturgesch. (A) 80(6): 32. Holotype: , Java: Semarang (HNHM).

Distribution: Vietnam (Dak Lak), Indonesia, Malaysia, New Guinea, Singapore, Sri Lanka, Thailand, the Philippines (Pham et al., 2011c).

Xanthopimpla konowi Krieger, 1899. Sitzber. Natur. Gesell. Leipzig, 1897/98: 87. Holotype: 9 , Japan (ZMHB).

Distribution: Vietnam (Lai Chau, Bac Can, Vinh Phuc, Ha Noi, Hoa Binh), China, India, Indonesia, Japan, Myanmar, Malaysia, Thailand (Townes \& Chiu, 1970).

Xanthopimpla leviuscula Krieger, 1914

Xanthopimpla leviuscula Krieger, 1914. Arch. f. Naturgesch., (A) 80(6): 45, 79. Holotype: $\bigcirc$, Vietnam: Tonkin [now northern Vietnam], Than-Moi (ZMHB).

Distribution: Vietnam (Ha Noi, Hoa Binh, Ha Tinh, Quang Binh, Thua Thien Hue, Ninh Thuan, Dong Nai), China, Laos, Myanmar, the
Philippines (Krieger, 1914).

Xanthopimpla melanacantha Krieger, 1914

Xanthopimpla melanacantha Krieger, 1914. Arch. f. Naturgesch. (A) 80(6): 22. Holotype: q, Thailand: Puket (ZMHB).

Distribution: Vietnam (Ha Tinh, Dong Nai),Indonesia, Malaysia, Thailand, the Philippines (Pham et al., 2011c).

Xanthopimpla minuta Cameron, 1905

Xanthopimpla minuta Cameron, 1905. Spolia Zeylanica, 3: 137. Holotype: + , Sri Lanka: Kandy (BMNH).

Distribution:Vietnam (Phu Tho, Nghe An, Quang Tri, Thua Thien-Hue, Dak Lak), China, India, Malaysia, Nepal, Sri Lanka, Taiwan, Thailand, the Philippines (Townes \& Chiu, 1970).

Xanthopimpla modesta (Smith, 1859)

Pimpla modesta Smith, 1859. Jour. Proc. Linn. Soc. London Zool., 5: 64. Holotype: $q$, Celebes: Macassar (OUMNH).

Distribution: Vietnam (Bac Can, Thai Nguyen, Vinh Phuc, Ha Noi, Hoa Binh, Ninh Binh, Nghe An, Thua Thien-Hue), China, Indonesia, Japan, Malaysia, Singapore, Taiwan, Thailand, the Philippines (Pham, 1997).

Remarks: This species has been recorded as a parasitoid of Chilo suppresalis (Lepidoptera: Pyralidae) on rice (Khuat \& Pham, 2007).

Xanthopimpla morsei Pham, Broad, Matsumoto \& Wägele, 2011

Xanthopimpla morsei Pham, Broad, Matsumoto \& Wägele, 2011. Zootaxa, 3056: 32. Holotype: $q$, Vietnam: Dong Nai, Cat Tien NP (RMNH).

Distribution: Vietnam (Dong Nai) (Pham et al., 2011c).

Xanthopimpla naenia Morley, 1913

Xanthopimpla naenia Morley, 1913. Faun. British India, Hymenoptera, 3(1): 115. Holotype: + , India (OUMNH).

Distribution: Vietnam (Lam Dong), India, Malaysia, Japan, Taiwan, the Philippines (Townes \& Chiu, 1970). 


\section{Xanthopimpla nana Schulz, 1906}

Xanthopimpla parva Cameron, 1905. Spolia Zeylanica 3: 136. Holotype: $\widehat{O}$, Sri Lanka: Peradeniya (BMNH). Junior homonym of Xanthopimpla parva Krieger, 1899.

Xanthopimpla nana Schulz, 1906. Spolia Hymenopterologica: 114. Replacement name.

Distribution: Vietnam (Thai Nguyen, Phu Tho, Ha Noi, Hoa Binh, Ninh Binh, Nghe An, Lam Dong, Khanh Hoa, Dong Nai), Cambodia, China, India, Indonesia, Nepal, Sri Lanka, Taiwan, Thailand, the Philippines (Pham et al., 2011c).

Xanthopimpla nigritarsis Cameron, 1903

Xanthopimpla nigritarsis Cameron, 1903. Jour. Straits Branch Roy. Asiatic Soc. 39: 138. Lectotype: + , Malaysia: Sarawak, Kuching (BMNH).

Distribution: Vietnam (Nghe An, Kon Tum, Dong Nai), India, Indonesia, Malaysia, the Philippines (Pham et al., 2011c).

Xanthopimpla ochracea (Smith, 1858)

Pimpla ochracea Smith, 1858. Jour. Proc. Linn. Soc. London Zool., 4: 172. Holotype: + , Indonesia: Molucca Island, Aru (OUMNH).

Xanthopimpla ochracea: Krieger (1899).

Distribution: Vietnam (Lai Chau, Ninh Binh, Phu Tho, Dak Lak), Australia, China, India, Indonesia, Myanmar, Papua New Guinea, Taiwan, Thailand, the Philippines (Pham et al., 2011c).

Xanthopimpla omega Pham, Broad, Matsumoto \& Wägele, 2011

Xanthopimpla omega Pham, Broad, Matsumoto \& Wägele, 2011. Zootaxa, 3056: 28. Holotype: $\hat{\delta}$, Vietnam: Ninh Binh, Cuc Phuong NP (IEBR).

Distribution: Vietnam (Ninh Binh) (Pham et al., 2011c).

Xanthopimpla oriole Pham, Broad, Matsumoto \& Wägele, 2011

Xanthopimpla oriole Pham, Broad, Matsumoto \& Wägele, 2011. Zootaxa, 3056: 36. Holotype: $q$, Vietnam: Thua Thien-Hue, Phong Dien NR (RMNH).
Distribution: Vietnam (Thua Thien-Hue) (Pham et al., 2011c)

Xanthopimpla panthera Pham, Broad, Matsumoto \& Wägele, 2011

Xanthopimpla panthera Pham, Broad, Matsumoto \& Wägele, 2011. Zootaxa, 3056: 37. Holotype: + , Vietnam: Dong Nai, Cat Tien NP (RMNH).

Distribution: Vietnam (Dong Nai) (Pham et al., 2011c).

Xanthopimpla pedator (Fabricius, 1775)

Ichneumon pedator Fabricius, 1775. Syst. Ent.: 828. Holotype: 옹 India (HMUG).

Distribution: Vietnam (Bac Can, Vinh Phuc, Ha Noi, Hoa Binh, Ninh Binh, Nghe An, Ha Tinh, Thua Thien-Hue), Bangladesh, China, India, Indonesia, Japan Malaysia, Myanmar, Pakistan, Singapore, Taiwan, the Philippines (Townes \& Chiu, 1970).

Remarks: This specieswas reared from a species of Erebidae(Lepidoptera) on lychee (Pham et al., 2011c). 1970

Xanthopimpla platyura Townes\& Chiu,

Xanthopimpla platyura Townes \& Chiu, 1970. Mem. Amer. Ent. Inst. 14: 199. Holotype: , Devala,Nilgiri Hills, south India (CNC).

Distribution: Vietnam (Ninh Binh), India, Indonesia (Townes \& Chiu, 1970).

Xanthopimpla pleuralis Cushman, 1925

Xanthopimpla pleuralis Cushman, 1925. Ent. Mitt., 14: 49. Holoype: $\widehat{~}$, Taiwan: Kangkou [= Kankau], Hengchun (DEI).

Distribution: Vietnam (Yen Bai, Bac Can, Thai Nguyen, Phu Tho, Hoa Binh, Nghe An, Ha Tinh), China, Indonesia, Nepal, Taiwan, the Philippines (Pham \& Le, 2007).

Xanthopimpla pleuroschista Townes \& Chiu, 1970

Xanthopimpla pleuroschista Townes \& Chiu, 1970. Mem. Amer. Ent. Inst, 14: 232. Holotype: + India: Khasi Hill (GPTA).

Distribution:Vietnam (Vinh Phuc), India (Townes \& Chiu, 1970). 
Xanthopimpla polyspila Cameron, 1907

Xanthopimpla polyspila Cameron, 1907. Tijdschr. v. Ent., 50: 101. Hololype: , India: Sikkim (BMNH).

Distribution: Vietnam (Thai Nguyen, Vinh Phuc, Ha Noi, Nghe An, Ha Tinh, Thua ThienHue, Dong Nai), China, India, Indonesia, Taiwan (PPRI, 1976).

Xanthopimpla porrecta Pham, Broad, Matsumoto \& Wägele, 2011

Xanthopimpla porrecta Pham, Broad, Matsumoto \& Wägele, 2011. Zootaxa, 3056: 29. Holotype: $q$, Vietnam: Bac Can, Ba Be NP (IEBR).

Distribution: Vietnam (Bac Can) (Pham et al., 2011c).

Xanthopimpla pseudosternata Pham, Broad, Matsumoto \& Wägele, 2011

Xanthopimpla pseudosternata Pham, Broad, Matsumoto \& Wägele, 2011. Zootaxa, 3056: 24. Holotype: $q$, Vietnam: Dak Lak, Chu Yang Sin NP (RMNH).

Distribution: Vietnam (Dak Lak) (Pham et al., 2011c). 1970

Xanthopimpla pulvinaris Townes \& Chiu,

Xanthopimpla pulvinaris Townes \& Chiu, 1970. Mem. Amer. Ent. Inst., 14: 271. Holotype: $q$, Taiwan: Shinchu (TARI).

Distribution: Vietnam (Phu Tho, Dak Lak), China, Laos, Malaysia, Taiwan, Thailand (Pham et al., 2011c).

Xanthopimpla punctata (Fabricius, 1781)

Ichneumon punctatus Fabricius, 1781. Species Insectorum, 1: 437. Holotype: $\widehat{\jmath}$, India: Coromadel (ZMUC).

Distribution: Vietnam (throughaut country), Afghanistan, Australia, China, India, Indonesia, Japan, Pakistan, Bangladesh, Guam, Laos, Malaysia, Mauritius, Myanmar, Nepal, Nigeria, Papua New Guinea, Russia, Sri Lanka, Singapore, Taiwan, Thailand, the Philippines, Togo (Townes \& Chiu, 1970).

Remarks: This speciesis known as a parasitoid of lepidopteran pupae: Cnaphalocrocis medinalis (Crambidae), Parnara guttata (Hesperiidae), Naranga aenescens (Noctuidae) on rice and of Lamprosema indica (Pyralidae) on soybean (Bui, 1990; Khuat \& Pham, 2007; Vu, 2007).

Xanthopimpla punctatissima Pham, Broad, Matsumoto \& Wägele, 2011

Xanthopimpla punctatissima Pham, Broad, Matsumoto \& Wägele, 2011. Zootaxa, 3056: 11. Holotype: $\delta$, Vietnam: Dong Nai, Cat Tien NP (RMNH)

Distribution: Vietnam (Dong Nai) (Pham et al., 2011c).

Xanthopimpla quatei Townes \& Chiu, 1970

Xanthopimpla quatei Townes \& Chiu, 1970. Mem. Amer. Ent. Inst., 14: 150. Holotype: + , Vietnam: $30 \mathrm{~km}$ north of Pleiku [now in Gia Lai Province], South Vietnam (BPBM).

Distribution: Vietnam (Gia Lai), Malaysia (Townes \& Chiu, 1970).

\section{Xanthopimpla regina Morley, 1913}

Xanthopimpla regina Morley, 1913. Faun. British India, Hymenoptera, 3(1): 118. Holotype: ${ }_{+}$, East Pakistan: Sylhet (BMNH).

Distribution: Vietnam (Bac Can, Thai Nguyen, Vinh Phuc, Phu Tho, Hoa Binh, Ninh Binh, Dak Lak), Bangladesh, China, India, Indonesia, Laos, Malaysia, Myanmar, Nepal, Pakistan, Singapore, Taiwan, Thailand (Pham et al., 2011c).

Xanthopimpla reicherti Krieger, 1914

Xanthopimpla reicherti Krieger, 1914. Arch. f. Naturgesch., (A) 80(6): 40, 89. Lectotype: + , Myanmar: Pekon on Loikaw River, Karenni State (ZMHB).

Distribution:Vietnam (Lao Cai, Hoa Binh, Ninh Binh, Nghe An), China, India, Myanmar, Thailand (Pham et al., 2011c).

Xanthopimpla sexlineata Cameron, 1907

Xanthopimpla sexlineata Cameron, 1907. Tijdschr. v. Ent. 50: 103. Holotype: $\hat{\jmath}$, India: Sikkim (BMNH).

Distribution: Vietnam (Kon Tum), India, Thailand (Pham et al., 2011c). 
Xanthopimpla spicula Pham, Broad, Matsumoto \& Wägele, 2011

Xanthopimpla spicula Pham, Broad, Matsumoto \& Wägele, 2011. Zootaxa, 3056: 12. Holotype: + , Vietnam: Dak Lak, Ea So NR (IEBR).

Distribution: Vietnam (Dak Lak) (Pham et al., 2011c).

Xanthopimpla sikkimensis Cameron, 1907

Xanthopimpla sikkimensis Cameron, 1907. Tijdschr. v. Ent., 50: 100. Holotype: , India: Sikkim (BMNH).

Distribution:Vietnam (Lai Chau, Hoa Binh, Ha Tinh, Thua Thien-Hue), India, Myanmar (Townes \& Chiu, 1970).

Xanthopimpla stemmator (Thunberg, 1822)

Ichneumon stemmator Thunberg, 1822. Mem. Acad. Imp. Sci., St. Peterbourg, 8: 262. Holotype: $\widehat{\partial}$, China (UUZM).

Distribution: Vietnam (Thai Nguyen, Phu Tho, Ha Noi, Hoa Binh, Thanh Hoa, Nghe An, Dong Nai, Tien Giang), China, India, Indonesia, Japan, Laos, Malaysia, Mauritius, Pakistan, Singapore, Sri Lanka, South Africa, Taiwan, Thailand, the Philippines (PPRI, 1976).

Remarks: In Vietnam, this species was reared from pupae of Chilo suppresalis (Pyralidae) on rice (Pham, 1997) and recently recorded as parasitoid of the corn borer Ostrinia furnacalis (Crambidae). Extralimitally, it has been extensively studied for its potential as a biocontrol agent against lepidopteran pests of rice (Townes \& Chiu, 1970).

Xanthopimpla sticta Townes \& Chiu, 1970

Xanthopimpla sticta Townes \& Chiu, 1970. Mem. Amer. Ent. Inst, 14: 53. Holotype: Indonesia: Sandakan, Borneo (USNM).

Distribution: Vietnam (Ninh Binh, Nghe An, Ha Tinh), Malaysia, Singapore, Thailand (Pham et al., 2011c).

Xanthopimpla transmaculata Wang \& Huang, 1993

Xanthopimpla transmaculata Wang \& Huang, 1993. Animals of Longqi Mountain: 729. Holotype: ㅇ, China: Mt. Longqi, Fujian
(IZCAS).

Distribution: Vietnam (Thua Thien-Hue), China (Pham et al., 2011c)

Xanthopimpla trias Townes \& Chiu, 1970

Xanthopimpla trias Townes \& Chiu, 1970. Mem. Amer. Ent. Inst., 14: 242. Holotype: India: Mercara, Mysore (GPTA).

Distribution: Vietnam (Vinh Phuc, Dak Lak), China, India, Nepal, Taiwan, Thailand (Townes \& Chiu, 1970).

Xanthopimpla tricapus Townes \& Chiu, 1970

Xanthopimpla tricapus Townes \& Chiu, 1970. Mem. Amer. Ent. Inst., 14: 260.

Xanthopimpla tricapus impressa Townes \& Chiu, 1970. Mem. Amer. Ent. Inst., 14: 260. Holotype: 9 , Myanmar: Toungoo, Karenni (ZMHB).

Distribution: Vietnam (Vinh Phuc, Nghe An, Dak Lak, Dong Nai), India, Indonesia, Malaysia, Myanmar, Thailand, the Philippines (Pham et al., 2011c).

Xanthopimpla varimaculata Cameron, 1907

Xanthopimpla varimaculata Cameron, 1907. Tijdschr. v. Ent., 50: 103. Holotype: $\widehat{o}^{\top}$, India: Sikkim (BMNH).

Distribution: Vietnam (Vinh Phuc, Hoa Binh, Nghe An, Ha Tinh, Thua Thien-Hue, Dak Lak, Dong Nai), China, India (Pham et al., 2011c).

\section{Subfamily RHYSSINAE Morley, 1913}

Epirhyssa Cresson, 1865

Epirhyssa tonkinensis Mocsary, 1905

Epirhyssa tonkinensis Mocsary, 1905. Ann. Mus. Natl. Hungarici, 3: 16. Holotype: ㅇ, North Vietnam: Mauson Mts., 2000-3000 ft, (HNHM).

Distribution: Vietnam (Lang Son) (Townes et al., 1961).

Lytarmes Cameron, 1899

(*) Lytarmes maculipennis (Smith, 1858)

Rhyssa maculipennis Smith, 1858. Jour. of Proc. Linn. Soc. London, Zool. 2: 120. Lectotype: $\uparrow$, Singapore (OUMNH). 
Distribution: Vietnam (Son La, Ninh Binh, Ha Tinh), India, Indonesia, Malaysia, Myanmar, Singapore (Kamath \& Gupta, 1972).

Megarhyssa Ashmead, 1900

Megarhyssa praecellens(Tosquinet, 1889)

Thalessa praecellens Tosquinet, 1889. Ann. Soc. Ent. Belgique, 33: 134. Holotype: , Russia: Wladiwostok.

Distribution: Vietnam (imprecise locality), China, Korea, Laos, Russia, Taiwan (Kamath \& Gupta, 1977).

\section{Subfamily STILBOPINAE Townes \&} Townes, 1949

Stilbops Forster, 1869

Subgenus Neostilbops Kasparyan, 1999

Stilbops (Neostilbops) gorokhovi Kasparyan, 1999

Stilbops (Neostilbops) gorokhovi Kasparyan, 1999. Ent. Obozrenie., 78(1): 382. Holotype: $q$, Vietnam: Gia Lai (ZIN).

Distribution: Vietnam (Gia Lai) (Kasparyan, 1999).

\section{Subfamily TERSILOCHINAE Schmiedeknecht,} 1910

Allophrys Forster, 1869

Allophrys occipitata Khalaim, 2011

Allophrys occipitata Khalaim, 2011. Zoosyst. Rossica, 20(1): 104. Holotype: o, Vietnam: Hoa Binh (ZIN).

Distribution: Vietnam (Hoa Binh), India (Khalaim, 2011).

Diaparsis Forster, 1869 2005

Diaparsis (Diaparsis) convexa Khalaim,

Diaparsis convexa Khalaim, 2005. Ent. Obozrenie, 84(2): 544. Holotype: +, Russia: Primorskii (ZIN)

Distribution: Vietnam (Hoa Binh), Russia (Khalaim, 2011).

Diaparsis (Diaparsis) dediticia Khalaim, 2011

Diaparsis (Diaparsis) dediticia Khalaim, 2011. Zoosyst. Rossica, 20(1): 117. Holotype:
, Vietnam: Vinh Phuc, Tam Dao NP (ZIN).

Distribution: Vietnam (Vinh Phuc, Hoa Binh, Ha Noi), Brunei (Khalaim, 2011).

Diaparsis (Diaparsis) hilaris Khalaim, 2011

Diaparsis (Diaparsis) hilaris Khalaim, 2011. Zoosyst. Rossica, 20(1): 118. Holotype: , Vietnam: Hoa Binh (ZIN).

Distribution: Vietnam (Hoa Binh) (Khalaim, 2011) 2011

Diaparsis (Diaparsis) minuta Khalaim,

Diaparsis (Diaparsis) minuta Khalaim, 2011. Zoosyst. Rossica, 20(1): 124. Holotype: , Vietnam: Hoa Binh (ZIN).

Distribution: Vietnam (Vinh Phuc, Hoa Binh) (Khalaim, 2011).

Diaparsis (Diaparsis) viela Khalaim, 2011

Diaparsis (Diaparsis) viela Khalaim, 2011. Zoosyst. Rossica, 20(1): 131. Holotype: o, Vietnam: Vinh Phuc, Tam Dao NP (ZIN).

Distribution: Vietnam (Vinh Phuc), Laos (Khalaim, 2011). 2011

Diaparsis (Diaparsis) vietnamica Khalaim,

Diaparsis (Diaparsis) vietnamica Khalaim, 2011. Zoosyst. Rossica, 20(1): 132. Holotype: , Vietnam: Hoa Binh (ZIN).

Distribution: Vietnam (Hoa Binh) (Khalaim, 2011). 2011

Diaparsis (Diaparsis) zispina Khalaim,

Diaparsis (Diaparsis) zispina Khalaim, 2011. Zoosyst. Rossica, 20(1): 133. Holotype: +, Vietnam: Hoa Binh (ZIN).

Distribution: Vietnam (Hoa Binh) (Khalaim, 2011)

Probles Forster, 1869

Subgenus Euporizon Horstmann, 1971

Probles (Euporizon) vietnamica Khalaim, 2011

Probles (Euporizon) vietnamica Khalaim, 2011. Zoosyst. Rossica, 20(1): 136. Holotype: , Vinh Phuc: Tam Dao NP (ZIN). 
Distribution: Vietnam (Vinh Phuc), China, Russia (Khalaim, 2011).

Sathropterus Forster, 1869

Sathropterus seduncus Khalaim, 2011

Sathropterus seduncus Khalaim, 2011. Zoosyst. Rossica, 20(1): 139. Holotype: + , Vinh Phuc: Tam Dao NP (ZIN).

Distribution: Vietnam (Vinh Phuc) (Khalaim, 2011).

\section{Subfamily XORIDINAE Shuckard, 1840}

Aplomerus Provancher, 1886

Aplomerus phamae Broad, 2014

Aplomerus phamae Broad, 2014. InVarga, Reshchikov \& Broad, Zootaxa, 3815(4): 595. Holotype: + Vietnam: Lam Dong, BidoupNulba NP (RMNH).

Distribution: Vietnam (Lam Dong) (Varga et al., 2014).

Xorides Latreille, 1809

Xorides elizabethae (Bingham, 1898)

Xylonomus elizabethae Bingham, 1898. Jour. Bombay Nat. Hist. Soc. 12: 116. Holotype: + , India: Sikkim, 1000-4000 ft. (ZMHB).

Distribution: Vietnam (imprecise locality), China, India, Indonesia, Malaysia, Myanmar, Thailand (Yu et al., 2012)

Xorides exquisitus (Tosquinet, 1903)

Moansa exquisitus Tosquinet, 1903. Mém. Soc. Ent. Belgique, 10: 57. Holotype: Indonesia: Java, Mt. Tengger, $4000 \mathrm{ft}$. (IRSNM).

Distribution: Vietnam (imprecise locality), China, Indonesia, Malaysia, Sri Lanka, the Philippines (Yu et al., 2012).

Xorides vitalisi (Turner, 1919)

Cyanoxorides vitalisi Turner, 1919. Ann. \& Mag. Nat. Hist. (9)4: 390. Holotype: +, Vietnam: Sapa (BMNH). 199).

Distribution: Vietnam (Lao Cai) (Turner,

\section{DISCUSSION}

In this paper 246 species of 18 subfamilies of Ichneumonidae are listed. Ten species are newly recorded here for the Ichneumonidae fauna of Vietnam. Certainly this checklist reveals only a small part of the rich diversity of this family in Vietnam.

Two genera, Xanthopimpla and Pimpla, are revealed as the most diverse genera with 60 and 10 species recorded, respectively. The information indicates the status of the study of ichneumonid fauna of Vietnam and of 25 ichneumonid species as parasitoids for the biological control of agricultural insect pests.

Acknowledgements: This research is funded by the Vietnam National Foundation for Science and Technology Development (NAFOSTED) under grant number 106-NN.05-2015.04. We would like to thank Dr Gavin Broad (The Natural History Museum, UK) for his responsible review, Dr Alexey Reshchikov (Sun Yat-sen University, China) for identifying two species of the Subfamily Ctenopelmatinae, Prof. Mao-ling Sheng (General Station of Forest Pest Management, China), Dr Eckhard Groll (Senckenberg Deutsches Entomologisches Institut, Germany), Dr Rikio Matsumoto (The Osaka Museum of Natural History, Japan), Dr Matthias Riedel (Oberammergau, Germany), and Nguyen Duc Hiep (Greifswald University, Germany)for providing scientific literatures. The first author thanks Idea Wild for supplying some equipment.

\section{REFERENCES}

Bennett A. M. R., 2001. Phylogeny of Agriotypinae (Hymenoptera: Ichneumonidae), with comments on the Subfamily relationships of the basal Ichneumonidae. Systematic Entomology, 26(3): 329-356.

Broad G. R., 2014. A revision of Sachtlebenia Townes, with notes on the species of Townesion Kasparyan (Hymenoptera: Ichneumonidae: Banchinae). Proceedsings of the Russian Entomological Society. St Petersburg, 85(1): 63-76.

Vu Quang Con, 1986. The peculiarities of formation of "host-parasite" systems on rice 
lepidopterouspests. Newsletter of Vietnam Academy of Science, 1: 55-62.

$\mathrm{Vu}$ Quang Con, 2007. The relationship of host and parasite of insects with focus on rice insect pests andtheir parasites in Vietnam. Science and Technology Publishing House: $278 \mathrm{pp}$.

Gauld I. D., Mitchell P. A., 1981. The taxonomy, distribution and host preferences of Indo-Papuan parasitic wasps of the Subfamily Ophioninae. Slough: CAB. Commonwealth Institute of Entomology, London: $611 \mathrm{pp}$.

Gupta V. K., Saxena K., 1987. A revision of the Indo-Australian species of Coccygomimus (Hymenoptera: Ichneumonidae). Oriental Insects, 21: 363-436.

Gupta V. K., Tikar D. T., 1976. Ichneumonologia Orientalis or a monographic study of Ichneumonidae of the Oriental Region, Part I. The tribe Pimplini (Hymenoptera: Ichneumonidae: Pimplinae). Oriental Insects Monograph, 1: 1-313.

Kamath M. K., Gupta V. K., 1972. Ichneumonologia Orientalis, Part II. The Tribe Rhyssini (Hymenoptera: Ichneumonidae). Oriental Insects Monograph, 2: 1-300.

Kasparyan D. R. 1999. New species of ichneumonid wasps of the Subfamily Stilbopinae (Hymenoptera, Ichneumonidae) of the Old World. Entomologicheskoe Obozrenie, 78(1): 163-173.

Kasparyan D. R., 2008. A new ichneumonid genus (Hymenoptera: Ichneumonidae: Mesochorinae) from Vietnam. Zoosystematica Rossica, 17(1): 85-87.

Khalaim A. I., 2011. Tersilochinae of South, Southeast and East Asia, excluding Mongolia and Japan (Hymenoptera: Ichneumonidae). Zoosystematica Rossica, 20: 96-148.

Pham Van Lam, 1997. A checklist of rice insect pests and their enemies in Vietnam. Agriculture Publishing House: 164 pp.

Pham Van Lam, 2002. Study on species composition of ichneumonid wasps (Hymenoptera: Ichneumonidae) in agricultural ecosystem. Proceedings of Plant Protection Research 2000-2002. Agriculture Publishing House: 150-154.

Khuat Dang Long, Pham Thi Nhi, 2007. An inventory of parasitic Ichneumonoid wasps (Hymenoptera: Ichneumonoidea) reared from agricultural pest insects in Vietnam. Proceedings of the 2nd National Workshop on Ecology and Biological Resources. Agricultural Publishing House, Ha Noi: 153-162.

Pham Thi Nhi, 2004. Mophological characteristics of two species of Metopius Panzer, 1806 (Hymenoptera: Ichneumonidae) in Vietnam. Journal of Biology, 26(3A): 70-72.

Pham Thi Nhi, Khuat Dang Long, 2007. Species composition of the Subfamily Metopiinae Forster, 1869 (Hymenoptera: Ichneumonidae) in Vietnam. Proceedings of the 2nd National Workshop on Ecology and Biological Resources. Agricultural Publishing House, Ha Noi: 197-208.

Pham N. T., 2013. Taxonomy and distributional pattern of Pimplinae (Hymenoptera: Ichneumonidae) from Vietnam. Ph.D. dissertation, Uni Bonn: 285 pp.

Pham Thi Nhi, Dang Thi Hoa, 2009. A new host record of Casinaria pedunculata Szepligeti (Hymenoptera: Ichneumonidae) from Vietnam. Proceedings of the $3^{\text {rd }}$ National Conference on Ecology and Biological Resources. Agricultural Publishing House, Ha Noi: 262-263.

Pham N. T., van Achterberg C., 2015. A review of the genus Phalgea Cameron (Hymenoptera: Ichneumonidae: Acaenitinae) with description of a new species from Vietnam. Zootaxa, 3947(1): 146-150.

Pham N. T., Broad G. R., Lampe K. H., 2010. Descriptions of two new species of the genus Augerella Gupta, 1962 (Hymenoptera: Ichneumonidae: Pimplinae) and the first record of $A$. orientalis (Gupta) 
from Vietnam. Zootaxa 2654: 17-29.

Pham N. T., Broad G. R., Lampe K. H., 2011 a. Descriptions of two new species of Augerella Gupta (Hymenoptera: Ichneumonidae: Pimplinae) from Vietnam. Zootaxa, 2745: 68.

Pham N. T., Broad G. R., Dang H. T., Böhme W., 2013a. A review of the genus Pimpla Fabricius, 1804 (Hymenoptera: Ichneumonidae: Pimplinae) from Vietnam with description of two new species. Organisms Diversity and Evolution, 13: 397-407.

Pham N. T., Broad G. R., Matsumoto R., Böhme W., 2012a. First record of the genus Acrodactyla Haliday (Hymenoptera: Ichneumonidae: Pimplinae) from Vietnam, with descriptions of six new species. Zootaxa, 3207: 40-53.

Pham N. T., Broad G. R., Matsumoto R., Wägele J. W., 2011b. Two new species of the genus Chablisea Gauld et Dubois (Hymenoptera: Ichneumonidae: Pimplinae) from Vietnam. Biologia, 66/6: 1134-1139.

Pham N. T., Broad G. R., Matsumoto R., Wägele J. W., 2011c. Revision of the genus Xanthopimpla Saussure (Hymenoptera: Ichneumonidae: Pimplinae) from Vietnam, with descriptions of fourteen new species. Zootaxa, 3056: 1-67.

Pham N. T., Broad G. R., Matsumoto R., Wägele W. J., 2012b. First record of the genus Brachyzapus Gauld and Dubois (Hymenoptera: Ichneumonidae: Pimplinae) from Vietnam, with descriptions of six new species. Journal of Natural History, 46(2728): 1639-1661.

Pham N. T., Broad G. R., Wägele J. W., 2011 d. The genus Acropimpla Townes (Hymenoptera: Ichneumonidae: Pimplinae) in Vietnam, with descriptions of three new species. Zootaxa, 2921: 1-12.

Pham N. T., Broad G. R., Wägele W. J., 2012c. A review of the genus Camptotypus Kriechbaumer (Hymenoptera, Ichneumonidae, Pimplinae) in Vietnam with description of a new species. Deutsche
Entomologische Zeitschrift, 59 (1): 129137.

Pham N. T., Broad G. R., Wägele W. J., 2013b. A review of the Theronia genus-group (Hymenoptera: Ichneumonidae: Pimplinae) from Vietnam with descriptions of five new species. Journal of Natural History. 47(2324): 1501-1538.

Pham N. T., Broad G. R., Wägele W. J., 2013c. Review of the genus Flavopimpla (Hymenoptera: Ichneumonidae: Pimplinae) from Vietnam with descriptions of two new species. Biologia, 64/4: 720-726.

Pham N. T., Broad G. R., Wägele W. J., 2013. First record of the genus Zaglyptus Förster (Hymenoptera: Ichneumonidae: Pimplinae) from Vietnam, with description of one new species. Zootaxa, 3702(5): 483-492.

Pham N. T., Broad G. R., Zwakhals K., 2012d. First record of the genus Dolichomitus Smith (Hymenoptera: Ichneumonidae) from Vietnam, with description of one new species. Zootaxa, 3519: 77-84.

Pham N. T., Khuat L. D., Dang H. T., 2011e. New records of Pimplinae (Hymenoptera: Ichneumonidae) from Vietnam. Proceedings of the 7th Vietnam National Conference on Entomology. Agricultural Publishing House, Ha Noi: 210-217.

Plant Protected Research Institute, 1976. Results of field survey on insects from 1967-1968. Agriculture Publishing House: $579 \mathrm{pp}$.

Reshchikov A., Kumar G., van Achterberg C., 2014. Review of Asiatic Neurogenia Roman, 1910(Hymenoptera, Ichneumonidae) with description ofthree new species. Tijdschrift voor Entomologie, 157(2014): 123-135.

Riedel M., 2011. Contribution to the Ichneumoninae (Hymenoptera, Ichneumonidae) of Southeastern Asia: 1. Tribes Clypeodromini, Listrodromini, Goedartini, Compsophorini, and Platylabini. Linzer Biologische Beitraege, 43(2):15491572. 
Riedel M., 2013. Contribution to the Ichneumoninae (Hymenoptera, Ichneumonidae) of Southeastern Asia: 2. Tribe Heresiarchini.Linzer Biologische Beitraege, 45(2): 2025-2076.

Takasuka K., Watanabe K., Yoshida H., 2012. New Records of Zatypota albicoxa (Hymenoptera, Ichneumonidae) and its Potential Host Spider Parasteatoda tepidariorum (Araneae, Theridiidae) from Vietnam. Japanese Journal of Systematic Entomology, 18(2): 447-450.

Townes H. K., Chiu S. C., 1970. The IndoAustralian species of Xanthopimpla (Ichneumonidae). Memoirs of the American Entomological Institute, 14: 1-372.

Uchida T., 1932. Beitrage zur Kenntnis der japanischen Ichneumoniden. Insecta Matsumurana, 6:145-168.

Townes H. K., Townes M., Gupta V. K., 1961.
A catalogue and reclassification of the Indo-Australian Ichneumonidae. Memoirs of the American Entomological Institute, $1: 1-522$

Varga O., Reshchikov A., Broad G. R., 2014. First record of the genus Aplomerus Provancher, 1886 (Hymenoptera: Ichneumonidae: Xoridinae) from the Oriental region, with descriptions of two new species. Zootaxa, 3815(4): 591-599.

Bui Tuan Viet, 1990. Ichneumonidae, pupae parasitoids of insect pests (Lepidoptera) on rice field in Ha Noi (composition, biology and ecology). $\mathrm{PhD}$ dissertation: 1-130.

Yu D. S., van Achterberg C., Horstmann K., 2012. Taxapad 2012, Ichneumonoidea 2011. Database on flash-drive. Ottawa, Ontario, Canada. Available from: http://www.taxapad.com (last accessed $5^{\text {th }}$ December 2016).

\section{DANH LỤC CÁC LOÀI ONG CỬ Ichneumonidae (Hymenoptera: Ichneumonoidea) ĐÃ GHI NHẬN Ở VIỆT NAM}

\section{Phạm Thị Nhị, Khuất Đăng Long}

Viện Sinh thái và Tài nguyên sinh vật, Viện Hàn lâm KH \& CN Việt Nam

\section{TÓM TẮT}

Nghiên cứu về khu hệ họ Ong cự Ichneumonidae (Hymenoptera: Icheumomnoidea) ở Việt Nam đã được tiến hành từ những năm đầu của thế kỷ $\mathrm{XX}$, tuy nhiên, những công bố về họ ong này xuất hiện rải rác trong các công trình nghiên cứu. Trong bài báo này, lần đầu tiên chúng tôi thống kê và tu chỉnh danh lục các loài ong cự đã được ghi nhận, đồng thời cung cấp các thông tin về phân bố trong và ngoài nước của chúng.

Theo số liệu thống kê, cho đến thời điểm hiện tại đã có 246 loài ong cự thuộc 87 giống, 18 phân họ được ghi nhận ở Việt Nam. Trong đó, một giống và 70 loài được mô tả dựa trên các mẫu chuẩn thu được ở Việt Nam. Trong danh lục này, bên cạnh tên chính thức của mồi loài, chúng tôi cung cấp thông tin về tên gốc, tài liệu mô tả gốc, nơi lưu giữ mẫu chuẩn và các tên đồng vật trước đây từng được sử dụng ở Việt Nam. Ngoài ra, ghi nhận mới 10 loài cho khu hệ ong cự của Việt Nam, bao gồm: Acerataspis clavata (Uchida, 1934); Charops brachypterus (Cameron, 1897); Chlorocryptus purpuratus (Smith, 1852); Chrionota townesi Uchida, 1957; Enicospilus hirayamai Uchida, 1955; Enicospilus melanocarpus Cameron, 1905; Lathrolestes roerichi Reschikov, 2011; Lytarmes maculipennis (Smith, 1858); Sachtlebenia sexmaculata Townes, 1963 và Stenichneumon appropinquans (Cameron, 1897). Mẫu vật của 10 loài ong cự này hiện đang được lưu giữ tại Viện Sinh thái và Tài nguyên sinh vật. Có 25 loài ong cự được ghi nhận là các loài ong ký sinh mà vật chủ của chúng là những sâu hại nguy hiểm cho các cây trồng nông nghiệp ở Việt Nam. Đây là cơ sở khoa học quan trọng cho các nghiên cứu tiếp theo về về họ Ichneumonidae ở Việt Nam.

Tù khóa: Họ Ong cự Ichneumonidae, côn trùng có ích, danh lục, ghi nhận mới, ong ký sinh. 